\title{
Minimal Influence of [NiFe] Hydrogenase on Hydrogen Isotope Fractionation in $\mathrm{H}_{2}$-Oxidizing Cupriavidus necator
}

\author{
Brian J. Campbell't, Alex L. Sessions ${ }^{2 *}$, Daniel N. Fox ${ }^{3}$, Blair G. Paul ${ }^{4}$, Qianhui Qin ${ }^{5}$, \\ Matthias Y. Kellermann ${ }^{6}$ and David L. Valentine ${ }^{1,4}$
}

OPEN ACCESS

Edited by: Jennifer Pett-Ridge,

Lawrence Livermore National Laboratory (DOE), United States

Reviewed by: William Lanzilotta,

University of Georgia, United States William D. Leavitt, Dartmouth College, United States Marcel Van Der Meer, Royal Netherlands Institute for Sea Research (NWO), Netherlands

${ }^{*}$ Correspondence:

Alex L. Sessions

als@gps.caltech.edu

†Present address:

Brian J. Campbell, Department of Environmental Quality,

Commonwealth of Virginia, Richmond, VA, United States

Specialty section:

This article was submitted to Microbiological Chemistry and Geomicrobiology, a section of the journal

Frontiers in Microbiology

Received: 06 October 2016 Accepted: 14 September 2017

Published: 04 October 2017

Citation: Campbell BJ, Sessions AL, FoX DN, Paul BG, Qin Q, Kellermann MY and Valentine DL (2017) Minimal Influence of [NiFe] Hydrogenase on Hydrogen Isotope Fractionation in $\mathrm{H}_{2}$-Oxidizing

Cupriavidus necator.

Front. Microbiol. 8:1886.

doi: 10.3389/fmicb.2017.01886

\begin{abstract}
${ }^{1}$ Department of Earth Science, University of California, Santa Barbara, Santa Barbara, CA, United States, ${ }^{2}$ Division of Geological and Planetary Sciences, California Institute of Technology, Pasadena, CA, United States, ${ }^{3}$ Undergraduate College of Letters and Sciences, University of California, Santa Barbara, Santa Barbara, CA, United States, ${ }^{4}$ Marine Science Institute, University of California, Santa Barbara, Santa Barbara, CA, United States, ${ }^{5}$ Interdepartmental Graduate Program in Marine Science, University of California, Santa Barbara, Santa Barbara, CA, United States, ${ }^{6}$ Institute for Chemistry and Biology of the Marine Environment, Carl von Ossietzky University, Wilhelmshaven, Germany
\end{abstract}

Fatty acids produced by $\mathrm{H}_{2}$-metabolizing bacteria are sometimes observed to be more D-depleted than those of photoautotrophic organisms, a trait that has been suggested as diagnostic for chemoautotrophic bacteria. The biochemical reasons for such a depletion are not known, but are often assumed to involve the strong D-depletion of $\mathrm{H}_{2}$. Here, we cultivated the bacterium Cupriavidus necator $\mathrm{H} 16$ (formerly Ralstonia eutropha $\mathrm{H} 16)$ under aerobic, $\mathrm{H}_{2}$-consuming, chemoautotrophic conditions and measured the isotopic compositions of its fatty acids. In parallel with the wild type, two mutants of this strain, each lacking one of two key hydrogenase enzymes, were also grown and measured. In all three strains, fractionations between fatty acids and water ranged from $-173 \%$ to $-235 \%$, and averaged $-217 \%$, $-196 \%$, and $-226 \%$, respectively, for the wild type, $\mathrm{SH}^{-}$mutant, and $\mathrm{MBH}^{-}$mutant. There was a modest increase in $\delta \mathrm{D}$ as a result of loss of the soluble hydrogenase enzyme. Fractionation curves for all three strains were constructed by growing parallel cultures in waters with $\delta D_{\text {water }}$ values of approximately $-25 \%$, 520\%, and 1100\%. These curves indicate that at least $90 \%$ of the hydrogen in fatty acids is derived from water, not $\mathrm{H}_{2}$. Published details of the biochemistry of the soluble and membrane-bound hydrogenases confirm that these enzymes transfer electrons rather than intact hydride $\left(\mathrm{H}^{-}\right)$ions, providing no direct mechanism to connect the isotopic composition of $\mathrm{H}_{2}$ to that of lipids. Multiple lines of evidence thus agree that in this organism, and presumably others like it, environmental $\mathrm{H}_{2}$ plays little or no direct role in controlling lipid $\delta \mathrm{D}$ values. The observed fractionations must instead result from isotope effects in the reduction of $\mathrm{NAD}(\mathrm{P}) \mathrm{H}$ by reductases with flavin prosthetic groups, which transfer two electrons and acquire $\mathrm{H}^{+}$(or $\mathrm{D}^{+}$) from solution. Parallels to NADPH reduction in photosynthesis may explain why $D / H$ fractionations in $C$. necator are nearly identical to those in many photoautotrophic algae and bacteria. We conclude that strong D-depletion is not a diagnostic feature of chemoautotrophy.

Keywords: Cupriavidus necator, hydrogenase, hydrogen isotope, D/H, fatty acid, autotrophic metabolism 


\section{INTRODUCTION}

The stable hydrogen isotope $(\mathrm{D} / \mathrm{H})$ ratios of $n$-alkyl lipids vary widely among the organisms that produce them. In plants and algae, fractionation between water and lipid hydrogen generally falls in a range of roughly $-130 \% 0$ to $-260 \%$ (Sessions et al., 1999; Chikaraishi et al., 2004; Zhang and Sachs, 2007; Chivall et al., 2014; Heinzelmann et al., 2015a). In heterotrophic microbes, the fractionation is typically smaller, and sometimes even reversed, leading to characteristically D-enriched lipids. Zhang et al. (2009a) further suggested that organisms using TCAcycle substrates would produce more D-enriched lipids than those using glycolytic substrates, and this pattern has been largely confirmed in a number of other species of aerobic heterotrophs (Dirghangi and Pagani, 2013; Dawson et al., 2015; Heinzelmann et al., 2015b; Kopf et al., 2015; Osburn et al., 2016).

In contrast, chemoautotrophs have been studied much less frequently. Valentine et al. (2004) reported on Sporomusa sp., an anaerobic $\mathrm{H}_{2}$-consuming acetogen that exhibits very large $\mathrm{D} / \mathrm{H}$ fractionations ( $>400 \%$ ) relative to water. Campbell et al. (2009) grew Desulfobacterium autotrophicum, an obligate anaerobe, on $\mathrm{H}_{2}+\mathrm{CO}_{2}$ or on formate and observed D-depletions of lipids generally between 260 and $390 \%$. Fatty acids in cultures grown on $\mathrm{H}_{2}$ were only slightly $(20-30 \%$ ) D-depleted relative to those grown on formate. Zhang et al. (2009a) presented data for Cupriavidus oxalaticus and C. necator grown aerobically on oxalate and formate, both of which lead to fractionations of 250-300\% . Heinzelmann et al. (2015b) grew Thiobacillus denitrificans on thiosulfate and measured fatty acids depleted in D by $217-275 \%$ relative to growth water. Osburn et al. (2011) presented data from a series of hot springs in Yellowstone National Park, in which $\mathrm{C}_{20}$ fatty acids were positively attributed to members of the order Aquificales. In this setting, Aquificales organisms are thought to grow as aerobic hydrogenotrophs, while their fatty acids exhibit $\mathrm{D}$-depletions relative to ambient water of $250-300 \%$.

Together these findings suggested a pattern in which chemoautotrophs are D-depleted relative to photoautotrophs, but by quite variable amounts. Larger depletions seemed to be generally associated with anaerobes and/or with hydrogenotrophy, although no mechanistic understanding of those patterns has yet been proposed. $\mathrm{H}_{2}$ is very strongly D-depleted relative to water at equilibrium, by $>600 \%$ (Horibe and Craig, 1995), providing a possible source for lower D/H ratios in hydrogenotrophs. Yet in the study of D. autotrophicum, Campbell et al. (2009) found that $\mathrm{H}_{2}$ contributed no hydrogen to lipids. Conversely, in cultures grown in $\mathrm{D}_{2} \mathrm{O}$, the hydrogenotrophic methanogen Methanothermobacter thermautotrophicus derives part of its methane hydrogen indirectly from $\mathrm{H}_{2}$ via the hydrogenase-catalyzed release of $\mathrm{H}^{+}$ into the intracellular aqueous proton pool (Spencer et al., 1980). It thus remains unclear whether - or why - hydrogenotrophy should be associated with D-depletion of lipids.

To study such issues, we cultivated three strains of $C$. necator aerobically on $\mathrm{H}_{2}+\mathrm{CO}_{2}$ : strain $\mathrm{H} 16$ wild type, a mutant lacking the membrane-bound hydrogenase $\left(\mathrm{MBH}^{-}\right)$, and a mutant lacking the soluble hydrogenase $\left(\mathrm{SH}^{-}\right)$. Both of these hydrogenases catalyze the reversible, heterolytic oxidation of $\mathrm{H}_{2}$ to $2 \mathrm{H}^{+}+2 e^{-}$and are involved in energy generation. These mutants allowed a direct test of the involvement of hydrogenase enzymes in generating strongly $\mathrm{D}$-depleted lipid signatures. We monitored the hydrogen isotopic composition of $\mathrm{H}_{2}$ during the growth of each culture and found that it shifted toward equilibrium with $\mathrm{H}_{2} \mathrm{O}$. Parallel cultures grown in waters of varying $\mathrm{D} / \mathrm{H}$ allowed us to indirectly assess the contributions of $\mathrm{H}_{2}$ and $\mathrm{H}_{2} \mathrm{O}$ to lipid hydrogen.

\section{MATERIALS AND METHODS}

\section{Bacterial Strains and Experimental Conditions}

Cupriavidus necator H16 (DSM 428, ATCC 17699) is a Gramnegative, aerobic, facultatively lithoautotrophic, $\mathrm{H}_{2}$-oxidizing bacterium that has been widely studied and reported under previous names, including Hydrogenomonas H16, Alcaligenes eutrophus (Davis et al., 1969), Ralstonia eutropha (Yabuuchi et al., 1995), and Wautersia eutropha (Vaneechoutte et al., 2004). Most recently, W. eutropha was reassigned to the prior taxon C. necator (Vandamme and Coenye, 2004).

C. necator H16 was obtained from the German Collection of Microorganisms and Cell Cultures (DSMZ), as were two mutant strains isolated by Pfitzner (1974): $\mathrm{SH}^{-}$(strain $\mathrm{LH}^{-}$7, DSM 416) and $\mathrm{MBH}^{-}$(strain $\mathrm{PH}^{-}$9, DSM 418) (Schink and Schlegel, 1978). All strains were grown in phosphate-buffered mineral media for chemolithotrophic growth (DSMZ Medium 81). In the main experiment, each strain was grown in three separate cultures having no, moderate $(\sim 500 \%)$ and high $(\sim 1100 \%)$ levels of $\mathrm{D}$-enrichment in the medium water. Culture media of differing $\mathrm{D} / \mathrm{H}$ ratio were prepared by volumetric dilution of a $10 \% \mathrm{D}_{2} \mathrm{O}$ stock to the required level (Campbell et al., 2009). To better understand variability, we conducted a second experiment in which C. necator $\mathrm{H} 16$ and the two hydrogenase mutants were cultivated in triplicate. These triplicate cultures of each strain were prepared without $\mathrm{D}$-enrichment of the medium water.

Culture conditions were chosen to balance the following objectives: (1) maximize biomass available for isotopic analysis, (2) minimize shifts in isotopic composition of water and $\mathrm{H}_{2}$, and (3) safely handle combustible mixtures of $\mathrm{H}_{2}+\mathrm{O}_{2}$. Cultures were therefore grown in moderate-sized batch cultures with headspace replenished once per day, as follows. The 200 -mL cultures were grown in $\sim 1050$-mL borosilicate glass bottles modified for closure with butyl rubber stoppers (13-mm inner diameter, 20-mm outer) and aluminum crimps. Before inoculation of sterile media, headspace gas was replaced with $\mathrm{H}_{2}+\mathrm{CO}_{2}$ (80:20 by volume). Gas was sterilized by flow through a sterile, cotton-packed syringe after removal of trace $\mathrm{O}_{2}$ by flow through a heated steel column containing reduced copper filings (Hungate, 1969). The $250 \mathrm{~mL}$ of filter-sterilized atmospheric air was added to the headspace via syringe, yielding a slight overpressure and headspace gas composition of approximately $62 \% \mathrm{H}_{2}, 15 \% \mathrm{CO}_{2}, 5 \% \mathrm{O}_{2}$, and $18 \% \mathrm{~N}_{2}$ (by volume). Headspace and liquid media were allowed to equilibrate overnight before inoculation. 
Starter cultures of each strain were grown to optical density $\geq 1(\mathrm{OD}, 660 \mathrm{~nm})$ in a single culture with $\delta \mathrm{D}_{\text {water }} \approx-25 \%$. To minimize the contribution of these cells to final biomass, they were diluted $\sim 10$-fold by transfer into sterile medium $\left(\delta \mathrm{D}_{\text {water }} \approx-25 \%\right.$ ). From this dilute culture, $1 \mathrm{~mL}$ was used to inoculate $200 \mathrm{~mL}$ sterile medium.

Cultures were incubated at $31^{\circ} \mathrm{C}$ with shaking at $100 \mathrm{rpm}$. Headspace gas composition was maintained by daily replacement with $\mathrm{H}_{2} / \mathrm{CO}_{2}$ and addition of air (as described above). OD was measured only once per day to minimize interference with the isotopic composition of headspace gases. The cultures of each strain were harvested when all three cultures exhibited $\mathrm{OD}>0.9$. Except where noted, collection and storage of samples for $\mathrm{D} / \mathrm{H}$ analyses followed the protocol of Campbell et al. (2009). Water samples were collected from each culture immediately after inoculation and immediately before harvest. A single gas sample of $\sim 10 \mathrm{~mL}$ was collected from each bottle immediately prior to harvest. In the first experiment, wild-type and $\mathrm{MBH}^{-}$cultures were harvested after collection of final $\mathrm{H}_{2}$ and water samples on the seventh day after inoculation. $\mathrm{SH}^{-}$cultures were similarly harvested on the 16th day after inoculation. In the second experiment, all cultures were harvested after collection of final $\mathrm{H}_{2}$ and water samples on the $23 \mathrm{rd}$ day after inoculation. For the first experiment, biomass was collected via vacuum filtration onto precombusted glass-microfiber filters (Whatman GF/F, $0.7-\mu \mathrm{m}$ nominal pore size). Filtration apparatus was soaked in $5 \% \mathrm{HCl}$ and rinsed thoroughly with milli-Q water between harvesting successive cultures. For the second experiment, biomass was pelleted by centrifugation and kept frozen prior to extraction.

\section{Fatty Acid and Polar Lipid Analyses}

Harvested biomass for fatty acid analyses was first lyophilized, then saponified in $1 \mathrm{M} \mathrm{KOH} / \mathrm{H}_{2} \mathrm{O}\left(70^{\circ} \mathrm{C}, 6 \mathrm{~h}\right)$ to provide the maximum yield of free fatty acids. $\mathrm{pH}$ was adjusted to $<2$ with $6 \mathrm{M} \mathrm{HCl}$, and the fatty acids were extracted into methyl t-butyl ether (Valentine et al., 2004). In the main experiment, a $10 \%$ aliquot of the extract from each sample was derivatized as the trimethylsilyl (TMS) ethers by reaction with bis-trifluoroacetamide (BSTFA) at $40^{\circ} \mathrm{C}$ for $15 \mathrm{~min}$, and analyzed by gas chromatography/mass spectrometry (GC/MS) on a Thermo Trace GC/DSQII MS. Fatty acid TMS ethers were identified from their mass spectra and retention times by comparison to library spectra and authentic standards. Double bond position for the 18:1 $\Delta 9$ fatty acid was confirmed by analysis of the picolinic ester, which fragments distinctively at the double bond position (Christie, 2003). The cyc-17:0, cyc-19:0, and $\mathrm{OH}-14: 0$ fatty acids were identified by comparison to an authentic standard of bacterial fatty acids (Sigma Chemical, St. Louis, MO, United States). Fatty acids were quantified as their TMS ethers by splitting $\sim 30 \%$ of the GC effluent to a flame ionization detector (FID). Samples were quantified relative to an internal standard (palmitic acid isobutyl ester) assuming equal response factors for all compounds. In the second experiment involving triplicate cultures in water without D-enrichment, fatty acids were derivatized and quantified as fatty acid methyl esters only. Hydroxyl-fatty acids were not quantified for this experiment.
To provide a more comprehensive understanding of membrane lipid composition, biomass from the second experiment was also subjected to polar lipid analysis. Biomass was first lyophilized and then aliquots were extracted according to a modified Bligh and Dyer protocol (Kellermann et al., 2016). In brief, a combination of lyophilized cell pellets and $3 \mathrm{~g}$ of pre-combusted silica sand were extracted in four cycles by ultrasonication using a solvent mixture (ratio 2:1:0.8; v:v:v) of methanol $(\mathrm{MeOH})$, dichloromethane (DCM), and aqueous buffer solution. A phosphate buffer (8.7 $\left.\mathrm{g} \mathrm{l}^{-1} \mathrm{KH}_{2} \mathrm{PO}_{4}, \mathrm{pH} 7.4\right)$ was used for the first two and a trichloroacetic acid buffer (50 $\mathrm{gl}^{-1}$ TCA, $\mathrm{pH} 2$ ) for the last two extraction steps. After each extraction, the solution was separated by centrifugation and all four supernatants were consecutively pooled in a separatory funnel, in which DCM and water had been added to facilitate optimal phase separation. After transfer of the organic phase, the remaining aqueous phase was extracted two more times with DCM. Finally, the pooled organic layers were washed three times with deionized milliQ water and the total lipid extract evaporated under a stream of $\mathrm{N}_{2}$ and stored at $-20^{\circ} \mathrm{C}$.

For polar lipid analysis, an aliquot of the total lipid extract was dissolved in MeOH:DCM, (9:1, v:v) and analyzed using a quadrupole time-of-flight mass spectrometer (Q-ToF-MS). Chromatographic separation was achieved on a Waters Acquity BEH C18 column $(1.7 \mu \mathrm{m}, 2.1 \times 150 \mathrm{~mm})$ with an ACQUITY Ultra Performance Liquid Chromatography (UPLC) H-Class System (Waters Co., Milford, MA, United States) coupled to a Synapt G2-Si HDMS high-resolution Q-ToF-MS (Waters Co., Manchester, United Kingdom) equipped with a LockSpray dual electrospray ion source operated in both positive (POS) and negative (NEG) ionization modes. The Q-ToF-MS was calibrated in resolution mode over a mass-to-charge $(\mathrm{m} / \mathrm{z})$ ranging from 50 to 2000 by using a $0.5 \mathrm{mmol} \mathrm{L}^{-1}$ sodium formate solution. For each run leucine enkephalin was used as lock mass, generating a reference ion for POS $\left([\mathrm{M}+\mathrm{H}]^{+}=556.2771\right)$ and NEG ionization mode $\left([\mathrm{M}-\mathrm{H}]^{-}=554.2615\right)$ to ensure a mass tolerance for all MS or MS/MS experiments of less than 1 ppm. Mass spectral data were collected using the $\mathrm{MS}^{\mathrm{e}}$ data acquisition function to simultaneously obtain information on the intact molecule (no collision energy applied) as well as their fragmentation data (collision energy ramp reaching from 15 to $75 \mathrm{eV})$.

Analytes were eluted at a flow rate of $0.4 \mathrm{ml} \mathrm{min}^{-1}$ using a linear gradient of $\mathrm{MeOH}: \mathrm{H}_{2} \mathrm{O}(85: 15, \mathrm{v}: \mathrm{v}$, eluent A) to $\mathrm{MeOH}$ :isopropanol (50:50, v:v, eluent B) both with $0.04 \%$ formic acid and $0.1 \%$ aqueous $\mathrm{NH}_{3}$ (Wörmer et al., 2013). The initial condition was $100 \%$ A held for $2 \mathrm{~min}$, followed by a gradient to $15 \% \mathrm{~B}$ in $0.1 \mathrm{~min}$ and a gradient to $85 \% \mathrm{~B}$ in $18 \mathrm{~min}$. The column was then washed with $100 \%$ B for 8 min and subsequently returned and held for $5 \mathrm{~min}$ to the initial conditions to equilibrate the column for the following run. The column temperature was set to $65^{\circ} \mathrm{C}$.

Lipid identification was achieved by analyzing the exact masses of possible precursor ions in positive and negative ionization modes, in combination with their characteristic fragmentation patterns and compound identities described in previous studies (i.e., Kellermann et al., 2011; Tavormina et al., 
2017). FA species of the individual polar lipids were identified in negative ionization mode. That is, after collisional activation of a polar lipid, the FA species are liberated with a neutral loss of water to yield an $[\mathrm{M}-\mathrm{H}-18]^{-}$ion. Note that the identification based on MS and MS/MS is a tentative characterization of the analytes. For comparison, all molecular ions (and their adducts) were quantified in positive ionization mode. Since response factors of individual lipid molecules were not accounted for, we only compare relative distribution of lipid species among samples.

\section{Hydrogen Isotopic Analyses}

$\mathrm{D} / \mathrm{H}$ ratios of water were analyzed with a pyrolysis elemental analyzer (ThermoElectron TC/EA) at UCSB as described by Campbell et al. (2009) and are reported as part-per-thousand (permil, or \%o) deviations from the VSMOW international standard in the conventional $\delta \mathrm{D}$ notation. $\delta \mathrm{D}$ values were calibrated by comparison to two NIST Standard Reference Materials (VSMOW, $\delta \mathrm{D}=0.0 \%$ and GISP, $\delta \mathrm{D}=-189.8 \% 0$ ) and three laboratory working standards of intermediate $\delta \mathrm{D}$ values. The root mean square (RMS) error of $\delta \mathrm{D}$ values for all analyzed standards $(n=48)$ was $3.3 \%$, which we interpret as the minimum uncertainty in measured values for $\delta \mathrm{D}_{\text {water }}$.

Fatty acids were further purified from the remaining (90\%) lipid extract by separation into four fractions by solid-phase extraction on an amino-propyl stationary phase following the methodology of Sessions (2006). Fatty acids (fraction 4) were then derivatized as methyl esters by reaction with $\mathrm{BF}_{3} / \mathrm{MeOH}\left(60^{\circ} \mathrm{C}\right.$ for $10 \mathrm{~min}$ ), and further reacted with acetic anhydride/pyridine $\left(80^{\circ} \mathrm{C}\right.$ for $\left.20 \mathrm{~min}\right)$ to produce the acetate derivatives of hydroxylfatty acids. $\mathrm{D} / \mathrm{H}$ ratios of fatty acid esters were determined by reference to both internal (coinjected) and external standard $n$-alkanes similar to those described by Campbell et al. (2009). The pooled standard deviation for replicate analyses of the external $n$-alkane standards was $1.4 \%$, and the RMS error was $3.3 \%(n=30)$. The pooled standard deviation for measurements of $n$-alkane standards coinjected with samples, was $3.4 \%$ o $(n=54)$. $\delta \mathrm{D}$ values of FAMEs were corrected for the contributions of methyl and acetyl groups as described by Valentine et al. (2004) and Campbell et al. (2009).

Samples of culture headspace gas for analysis of $\mathrm{H}_{2}$ isotope ratio were dried by passing over anhydrous $\mathrm{CaSO}_{4}$, then stored in glass serum bottles with butyl rubber stoppers until analysis, 197-206 days. Samples were introduced to the IRMS via a ThermoElectron GasBench device, with $\delta \mathrm{D}$ values measured directly on the $\mathrm{H}_{2}$ by comparison to standards (Campbell et al., 2009). Mean precision for unknown samples was $3.4 \%$.

\section{Isotope Data Analysis}

C. necator metabolizes two potential sources of hydrogen: water and $\mathrm{H}_{2}$. By varying the isotopic compositions of the inputs, and measuring the concomitant changes in isotopic composition of lipids, we can deduce the relative contributions of those two sources to lipid hydrogen. A complication, however, is that uptake/utilization of hydrogen from either $\mathrm{H}_{2}$ or $\mathrm{H}_{2} \mathrm{O}$ is likely accompanied by kinetic fractionations of substantial magnitude. It is not possible to uniquely deconvolve both the relative contributions of those two external sources, and the isotopic fractionations associated with each, using the experimental data available here (see discussion in Sessions and Hayes, 2005). Instead, we follow the approach of Zhang et al. (2009a) in constructing 'fractionation curves,' as follows.

Isotopic abundance data for lipids and water reported in delta notation by the Isodat software were first converted to isotope ratios using $R_{\mathrm{x}}=\left(\delta \mathrm{D}_{\mathrm{x}}+1\right) R_{\mathrm{std}}$, where $\delta \mathrm{D}_{\mathrm{x}}$ is the measured $\delta \mathrm{D}$ value of sample $x$ and $R_{\text {std }}$ is the isotope ratio of VSMOW: 0.00015576 (Hagemann et al., 1970). For each fatty acid, we then fit the data from parallel cultures that were grown in water of differing $\delta \mathrm{D}$ values to an equation (Sessions and Hayes, 2005) of the form.

$$
R_{\mathrm{FA}}=X_{\text {water }} \alpha_{\mathrm{FA} / \text { water }} R_{\text {water }}+\left(1-X_{\text {water }}\right) \alpha_{\mathrm{FA} / \mathrm{H} 2} R_{\mathrm{H} 2}
$$

where $R$ is the isotope ratio of fatty acids, growth water, and hydrogen gas, $\alpha$ is the net isotopic fractionation factor associated with utilization of each hydrogen source, and $X_{\text {water }}$ is the abundance-fraction of fatty acid hydrogen originating from water (as opposed to $\mathrm{H}_{2}$ ). Briefly, a linear regression of $R_{\mathrm{FA}}$ against $R_{\text {water }}$ yields a slope equal to $X_{\text {water }} \alpha_{\mathrm{FA}} /$ water and an intercept equal to $\left(1-X_{\text {water }}\right) \alpha_{\mathrm{FA} / \mathrm{H} 2} R_{\mathrm{H} 2}$. Since $R_{\mathrm{H} 2}$ is known, $\left(1-X_{\text {water }}\right) \alpha_{\mathrm{FA} / \mathrm{H} 2}$ can be computed directly. This leaves three unknowns $\left(X_{\text {water }}, \alpha_{\mathrm{FA} / \text { water }}, \alpha_{\mathrm{FA} / \mathrm{H} 2}\right)$ and only two constraints. The set of all possible solutions that satisfy the experimental constraints (for a given fatty acid under constant growth conditions) then define a curve in a plot of $\alpha_{\mathrm{FA} / \text { water }}$ versus $\alpha_{\mathrm{FA} / \mathrm{H} 2}$, which Zhang et al. (2009a) termed a 'fractionation curve.' Further inferences about the magnitudes of the individual fractionations can be drawn from this construct, as discussed in Sections "Results" and "Discussion."

\section{RESULTS}

\section{Culture Conditions and Growth}

Conditions for the nine independent cultures of the first experiment are summarized in Table 1. In cultures with low-D water, $\delta D_{\text {water }}$ increased slightly $(4-6 \% 0)$ over time, while the other treatments exhibited no significant change in $\delta D_{\text {water }}$. Culture media with the lowest $\delta D_{\text {water }}$ values were farthest from isotopic equilibrium with the supplied $\mathrm{H}_{2}$, and D-enrichment of water in these cultures would be consistent with hydrogenasecatalyzed isotopic exchange between $\mathrm{H}_{2}$ and $\mathrm{H}_{2} \mathrm{O}$ (see discussion in Campbell et al., 2009). For the purpose of estimating fractionations, we adopt the average of the two water $\delta \mathrm{D}$ measurements for each culture (reported in Table 1).

Values of $\delta \mathrm{D}_{\mathrm{H} 2}$ changed more dramatically between the last replacement of the headspace and the gas sample taken immediately before harvesting (a period of approximately 1 day). In every culture, $\delta \mathrm{D}_{\mathrm{H} 2}$ changed by several hundred permil in the direction of equilibrium with water (Table 1). The rate of isotopic change was also a function of the extent of $\mathrm{H}_{2}$ /water disequilibrium, consistent with isotopic exchange as the controlling mechanism for changes in $\delta \mathrm{D}$ values of both water and $\mathrm{H}_{2}$. Such shifts in $\delta \mathrm{D}_{\mathrm{H} 2}$ must have occurred following every replacement of headspace gas, albeit probably to lesser extents when there was less biomass present. These significant shifts in 
$\delta \mathrm{D}_{\mathrm{H} 2}$ complicate the analysis of isotopic fractionations, because the harvested biomass must integrate isotopic signals from the changing $\mathrm{H}_{2}$. Fortunately, as we show below, the contribution of $\mathrm{H}_{2}$ to lipid hydrogen is minor; thus, even these large shifts in $\delta \mathrm{D}_{\mathrm{H} 2}$ introduce only minor noise into values of $\delta \mathrm{D}_{\mathrm{FA}}$ (see section " $\delta \mathrm{D}$ Values of Fatty Acids").

Growth curves for the cultures were constructed from oncedaily measurements of OD, which offered limited resolution of the growth phases. The growth curves for all three strains in both rounds of experiment became pseudo-linear after a brief period of apparently exponential growth (Supplementary Table S1). This behavior is consistent with growth limited by $\mathrm{O}_{2}$ supply, which precludes calculation of meaningful generation times. Nevertheless, growth of the $\mathrm{SH}^{-}$mutant was appreciably slower than for the other two strains, as has been previously observed (Schink and Schlegel, 1978). Given that all strains received the same daily supply of $\mathrm{H}_{2}+\mathrm{O}_{2}$, this result presumably reflects a lower growth efficiency of the $\mathrm{SH}^{-}$strain, related to its reliance on reverse electron transport for $\mathrm{NAD}^{+}$reduction (discussed below).

Table 2 summarizes the conditions for the triplicate cultures of the second experiment, in which the three strains grew in medium water without D-enrichment. The results were similar to those of the first experiment, including impaired growth of the $\mathrm{SH}^{-}$strain relative to the others.

\section{Relative Abundances of Fatty Acids and Polar Lipids}

The predominant lipids in C. necator were $n$-alkyl fatty acids. In every strain and culture condition of the first experiment, the same six fatty acids accounted for $98 \%$ of the total abundance: tetradecanoic (14:0), 3-hydroxytetradecanoic (OH-14:0), hexadecanoic (16:0), 9,10-methylenehexadecanoic (cyc-17:0), octadec-9-enoic (18:1 $\Delta 9)$, and 9,10-methyleneoctadecanoic

TABLE 1 | Experimental conditions for growth of $C$. necator.

\begin{tabular}{|c|c|c|c|c|}
\hline \multirow[t]{2}{*}{$\delta D_{\text {water }}(\% 0)^{a}$} & \multicolumn{3}{|c|}{$\delta D_{\mathrm{H} 2}(\% 0)$} & \multirow[t]{2}{*}{ OD 660 at harvest } \\
\hline & Supplied & Final & Equilibriumb $^{b}$ & \\
\hline \multicolumn{5}{|l|}{ Wild type } \\
\hline$-25(1)^{\mathrm{C}}$ & $-180(1)$ & $-632(2)$ & -736 & 0.922 \\
\hline $523(2)$ & $-180(1)$ & $-469(1)$ & -587 & 0.938 \\
\hline 1106 (10) & $-180(1)$ & $-364(3)$ & -430 & 0.927 \\
\hline \multicolumn{5}{|l|}{$\mathrm{SH}^{-}$} \\
\hline$-24(1)$ & $-180(1)$ & $-591(2)$ & -736 & 0.964 \\
\hline $519(2)$ & $-180(1)$ & $-451(1)$ & -589 & 0.984 \\
\hline $1104(2)^{*}$ & $-180(1)$ & $-355(1)$ & -430 & 0.989 \\
\hline \multicolumn{5}{|l|}{$\mathrm{MBH}^{-}$} \\
\hline$-24(1)$ & $-180(1)$ & $-594(2)$ & -736 & 1.030 \\
\hline $524(2)$ & $-180(1)$ & NA & -587 & 1.042 \\
\hline 1110 (5) & $-180(1)$ & $-364(1)$ & -429 & 1.025 \\
\hline
\end{tabular}

${ }^{a}$ Reported $\delta D_{\text {water }}$ value is the average of measurements taken at start and end of culture growth. These values differed by $<9 \%$ in all cultures except one $\left(^{*}\right)$, where they differed by $17 \%$. b ${ }^{b}$ redicted $\delta D_{H_{2}}$ at isotopic equilibrium with supplied water, based on the equation of Horibe and Craig (1995). ' Values in parentheses are one standard deviation of replicate analyses of the same sample.
TABLE 2 | Summary of $\delta D$ values (mean, one standard deviation) from triplicate cultures grown in media without D-enriched water.

\begin{tabular}{lccc}
\hline C. necator strain: & Wild type & $\mathbf{S H}^{-}$ & $\mathbf{M B H}^{-}$ \\
\hline Water & & & \\
Initial & $-35(2)$ & $-37.4(0.7)$ & $-34(1)$ \\
Final & $-15(1)$ & $-18.6(0.6)$ & $-13(2)$ \\
Mean & -25 & -28.0 & -24 \\
H $_{2}$ & & & \\
Supplied & & $-181(2)$ & $-181(2)$ \\
Final & $-181(2)$ & $-449(13)$ & $-471(5)$ \\
Fatty acid & $-472(10)$ & & $-216(6)$ \\
14:0 & & $-211.7(0.2)$ & $\mathrm{NA}$ \\
3-OH14:0 & & NA & $-200(4)$ \\
16:0 & $-214(3)$ & $-192(4)$ & $-226(4)$ \\
cyc-17:0 & $-204(1)$ & $-207(3)$ & $-224(6)$ \\
18:1 $\Delta 9$ & $-229(3)$ & $-209(10)$ & $-219(9)$ \\
cyc-19:0 & $-232(4)^{\mathrm{c}}$ & -205 & -213 \\
Weighted mean & $-230(6)$ & -217 &
\end{tabular}

a Duplicate analyses of two separate samples of the gas supplied to all cultures. ${ }^{\mathrm{b}}$ Not analyzed (see text); included for continuity with Table 3. "Mass sufficient for isotope analysis in two of the three cultures $(n=2)$.

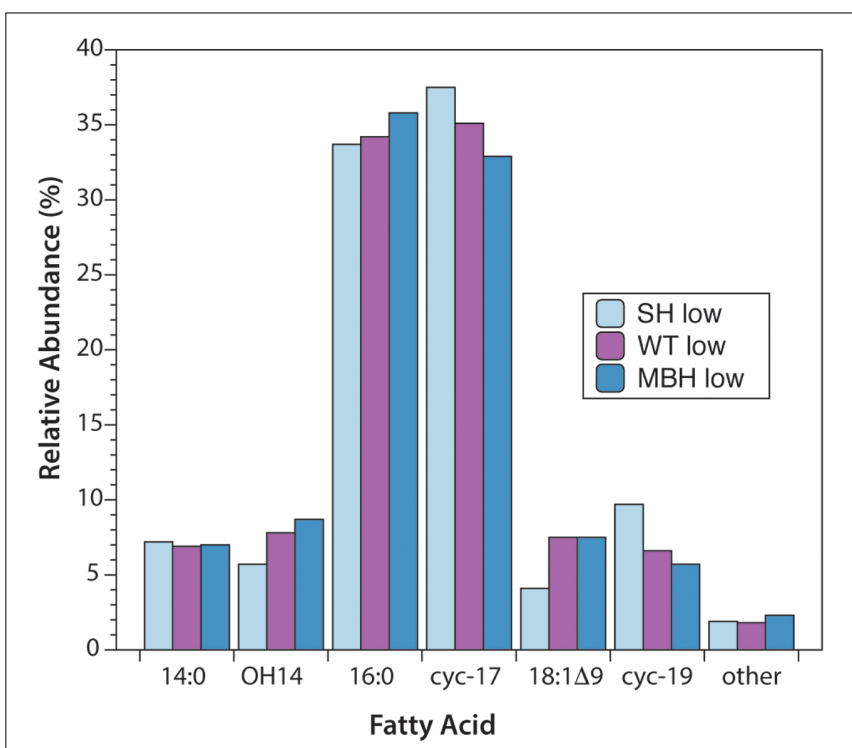

FIGURE 1 | Relative abundances of fatty acids from the three strains of C. necator, all grown in non-D-enriched medium. Colors represent the $\mathrm{SH}^{-}$ (light blue), wild-type (purple), and $\mathrm{MBH}^{-}$(dark blue) strains.

(cyc-19:0) acids (Figure 1). 16:0 and cyc-17:0 were the most abundant, accounting for $\sim 70 \%$ of total fatty acids. Variations in fatty acid abundance between culture conditions were insignificant, and between strains were minor. The biggest differences between strains were for 18:1 $\Delta 9$ (3.5\% in $\mathrm{SH}^{-}, 7.0 \%$ in the wild type, $7.6 \%$ in $\left.\mathrm{MBH}^{-}\right)$and for cyc-19:0 $\left(9.3 \%\right.$ in $\mathrm{SH}^{-}$, $6.5 \%$ in the wild type, $5.5 \%$ in $\mathrm{MBH}^{-}$).

The second experiment yielded the same abundant fatty acids, with the exception that $\mathrm{OH}-14: 0$, was not quantified (see section "Fatty Acid and Polar Lipid Analyses"). Relative abundances of 
the other five predominant fatty acids were comparable to those of the main experiment after exclusion of $\mathrm{OH}-14: 0$ from the total abundance. In the second experiment, on average across strains, 16:0 represented approximately $5 \%$ more, and 18:1 approximately $5 \%$ less, of the total abundance of the predominant fatty acids (excluding $\mathrm{OH}-14: 0$ ). The results were otherwise similar, with the average results for 14:0, cyc-17:0, and cyc-19:0 differing by less than $1 \%$ between experiments. Differences between strains were even smaller in the second experiment than in the first. These small differences in the relative abundance of fatty acids are unlikely to affect their $\mathrm{H}$-isotopic compositions. Complete abundance data are provided in the Supplementary Table S1.

The polar lipid composition among the analyzed C. necator strains (wild type, $\mathrm{MBH}^{-}$, and $\mathrm{SH}^{-}$) showed little variation. In all samples, the phospholipids were dominant and comprised $>60 \%$ of the polar lipid composition, followed by the electron and proton carrier ubiquinone (UQ) and two bacteriohopanols (BHPs; Figures 2A,B). The detected phospholipid headgroups were composed of only phosphatidylethanolamine (PE) and phosphatidylglycerol (PG; Figure 2C). However, PE clearly dominated at roughly $95 \%$ of the phospholipid fraction, with highest relative contributions containing the fatty acid combination of 16:0 and cyc-17:0, 16:0 and 16:0, and cyc17:0 and cyc-19:0 (Figure 2D). Furthermore, the results of this liquid chromatography (LC)-based fatty acid analysis resemble the relative proportions of fatty acids, except for $\mathrm{OH}-14: 0$, demonstrated by the GC-based analyses described above. LCbased results also indicate the predominance of 16:0 and cyc-17:0 fatty acids, which together represented greater than $80 \%$ of the total (Figure 2E).

Polar lipid analysis of biomass from the second experiment indicated that the most significant variation among the $C$. necator strains was an increase in the relative proportion of UQ in the $\mathrm{MBH}^{-}$mutant and foremost in the $\mathrm{SH}^{-}$mutant (Figure 2B). The contribution of cyc-17:0 also increased, and that of cyc19:0 decreased, in the same order among the strains (Figure 2E). Complete polar lipid data are provided in the Supplementary Table S1.

\section{$\delta$ D Values of Fatty Acids}

C. necator $\delta \mathrm{D}_{\mathrm{FA}}$ values ranged from $-260 \%$ to $686 \%$ (Figure 3, Tables 2, 3, and Supplementary Figure S1). The range for a given $\delta D_{\text {water }}$ treatment was far narrower, but increased with the $\delta \mathrm{D}$ of the water; i.e., $\delta \mathrm{D}_{\mathrm{FA}}$ values were more variable between D-enriched cultures. Analyses of triplicate cultures (second experiment) indicated that biological variability in these $\delta \mathrm{D}_{\mathrm{FA}}$ values was generally $<5 \%$, and always $<10 \%$ ( $1 \sigma)$. The pooled standard deviation of all fatty acids in all replicate cultures was $5.1 \%$, a value that we take as representative uncertainty in the following discussion. This is only slightly greater than analytical precision (typically $<4 \% 0$ ). In each strain and each culture condition of both experiments, fatty acids were strongly D-depleted relative to growth water, by $178-278 \%$, with the magnitude of this depletion increasing with $\delta D_{\text {water. In the }}$ first experiment (Table 3 and Supplementary Table S1), the abundance-weighted mean fractionation between fatty acids and growth water was $-217 \%$ in the wild type, $-196 \%$ in $\mathrm{SH}^{-}$, and $-226 \%$ in $\mathrm{MBH}^{-}$(all values for the $\delta \mathrm{D}_{\text {water }}=-25 \%$ growth condition). In the second experiment at similar growth conditions (Table 2 and Supplementary Table S1), abundanceweighted mean fractionations were all shifted to slightly heavier values: $-197 \%$ in the wild type, $-182 \%$ in $\mathrm{SH}^{-}$, and $-194 \%$ in $\mathrm{MBH}^{-}$. To test whether the small changes in abundance of 18:1 and cyc-19:0 FA between strains could be responsible for such differences, we recalculated weighted mean $\delta \mathrm{D}$ values excluding those two compounds. Differences between the strains remain unchanged when these fatty acids are excluded (not shown).

Comparing $\delta \mathrm{D}_{\mathrm{FA}}$ values within each culture, the $16: 0$ fatty acid was consistently the most D-enriched fatty acid, often by 20 $30 \%$ relative to other fatty acids. In eight of the nine cultures in the main experiment (Table 3), OH-14:0 was the second most D-enriched, and 18:1 $\Delta 9$ was the most D-depleted. For all fatty acids, $\delta \mathrm{D}$ values varied systematically among strains in the order $\mathrm{MBH}^{-}<$wild type $<\mathrm{SH}^{-}$, with the three strains typically spanning a range of 30-40\% . That range was broader for cultures grown in D-enriched medium.

The results of the second experiment (Table 2) confirmed the relative $\mathrm{D}$-enrichment of the 16:0 fatty acid, but $\mathrm{OH}$ 14:0 was not analyzed. These data also confirmed that fatty acids from the $\mathrm{SH}^{-}$strain were significantly more D-enriched than those from the other strains $(p<0.002)$, but did not yield consistent variation in $\delta \mathrm{D}_{\mathrm{FA}}$ between the $\mathrm{MBH}^{-}$mutant and the wild type. Accordingly, the differences between wild type and $\mathrm{MBH}^{-}$strains for the $\delta \mathrm{D}_{\text {water }}=-25 \%$ experiments are not statistically significant $(p<0.19)$. However, the second experiment included only cultures grown in low-D medium, whereas in the first experiment the difference in $\delta \mathrm{D}_{\mathrm{FA}}$ between the $\mathrm{MBH}^{-}$mutant and the wild type was stronger at higher levels of D-enrichment (see Supplementary Figure S1). Assuming the biological variance in high-D conditions is similar to that in the low-D experiment, i.e., that $\sigma_{\delta D}=5.1 \%$, the differences between $\mathrm{MBH}^{-}$and the wild type at medium- and high-D enrichments would be significant at $p<0.001$. Therefore, we retain for discussion the observed systematic variation in $\delta D_{\mathrm{FA}}$ in the order $\mathrm{MBH}^{-}<$wild type $<\mathrm{SH}^{-}$.

Fatty acid $\delta \mathrm{D}$ values were strongly correlated with those of water (Figure 4; $R^{2} \geq 0.9999$ and $p<0.003$ in every regression). Slopes for these correlations ranged from 0.686 to 0.783 , consistent with water providing the majority of hydrogen in fatty acids. For all three strains, slopes were highest for 16:0 fatty acid, and lowest for 18:1 $\Delta 9$ fatty acid. This order follows the pattern of fractionation (16:0 is D-enriched and so less fractionated, thus $\alpha$ is larger; $18: 1$ is D-depleted and so more fractionated, $\alpha$ is smaller), as would be expected if the proportion of water-derived hydrogen $\left(X_{\text {water }}\right)$ remained roughly constant across fatty acids. Similarly, for any given fatty acid, slopes were slightly higher in $\mathrm{SH}^{-}$and slightly lower in $\mathrm{MBH}^{-}$relative to the wild type, again following the pattern of fractionation $\left(\mathrm{SH}^{-}\right.$is $\mathrm{D}$-enriched and less fractionated; $\mathrm{MBH}^{-}$is $\mathrm{D}$-depleted and more fractionated).

Fractionation curves derived from these linear regressions (Figure 5) are virtually identical for the same fatty acid from different strains, including the hydrogenase mutants. In contrast, 

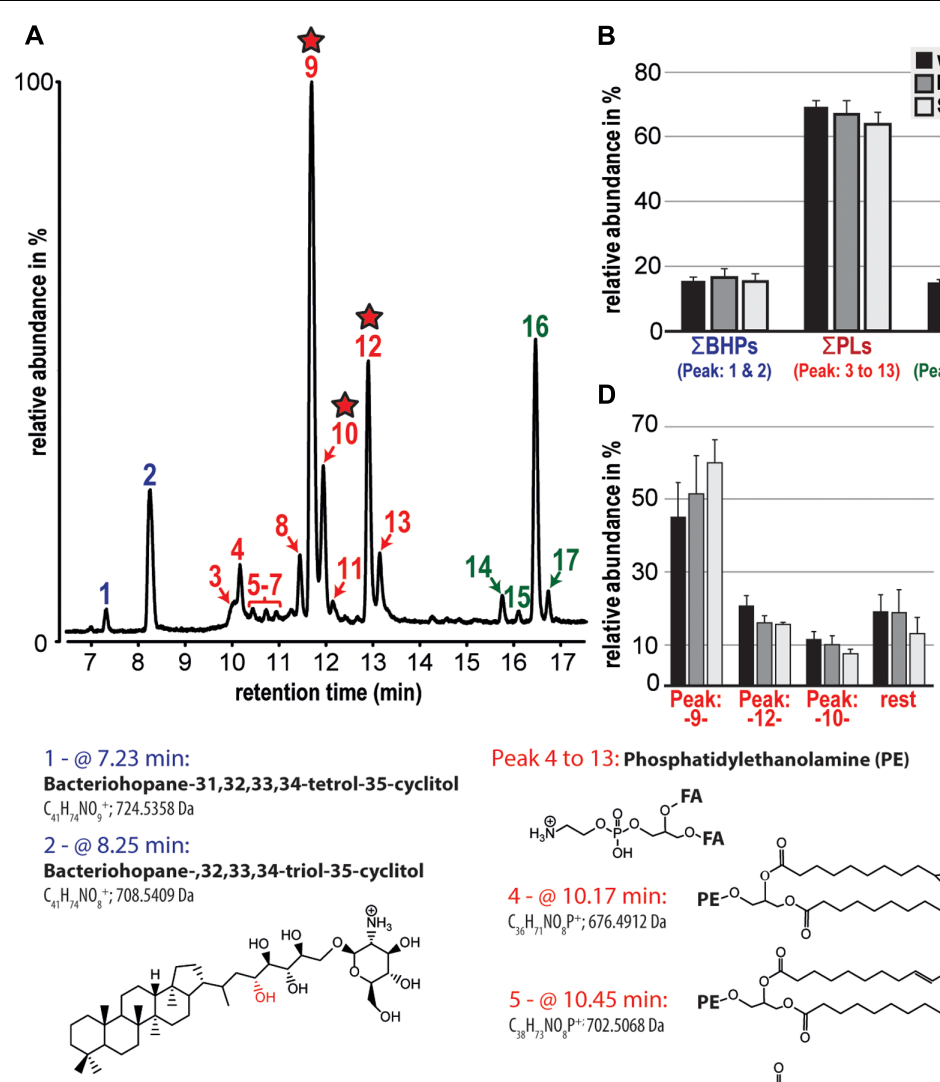

3 - @ 10.06 min:
Phosphatidylglycerol (PG)
Pho

$14 \& 16$ - @ $15.76 \& 16.47$ min: Ubiquinone 8 $\mathrm{C}_{49} \mathrm{H}_{75} \mathrm{O}_{4}^{+} ; 727.5660 \mathrm{Da}$<smiles>CCC(C)CCC(C)CCCCCCC(C)CCC(C)CCC(C)CCC(C)CCC(C)CCC(C)CCC(C)C</smiles>

15 - @ $16.11 \mathrm{~min}:$ 2-Octaprenylpheno $\mathrm{C}_{46} \mathrm{H}_{71} \mathrm{O}^{+} ; 639.5499 \mathrm{Da}$<smiles>CC(C)CCCC(C)CCCCCC(C)CCCC(C)CCC(C)CCC(C)CCC(C)CCC(C)CCC(C)C</smiles>

17 - @ 16.74 min: 2-Octaprenyl-6-methoxyphenol $\mathrm{C}_{44} \mathrm{H}_{73} \mathrm{O} ; 2 ; 669.5605 \mathrm{Da}$<smiles>CC(C)CCC(C)CCC(C)CCCC(C)CCC(C)CCC(C)CCC(C)CCC(C)CCC(C)CCC(C)C</smiles>

Peak 4 to 13: Phosphatidylethanolamine (PE)

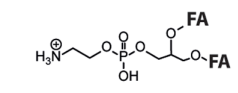

4 - @ 10.17 min: $\mathrm{C}_{30} \mathrm{H}_{71} \mathrm{NO}_{8} \mathrm{P}+; 676.4912 \mathrm{Da}$

5 - @ $10.45 \mathrm{~min}:$ $\mathrm{C}_{38} \mathrm{H}_{73} \mathrm{NO}_{8} \mathrm{P}+702.5068 \mathrm{Da}$

6 - @ $10.73 \mathrm{~min}:$ $\mathrm{C}_{33} \mathrm{H}_{73} \mathrm{NO}_{8} \mathrm{P}^{+} ; 690.5068 \mathrm{Da}$
FA

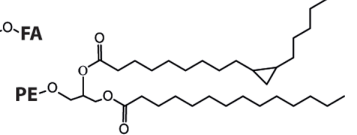

50

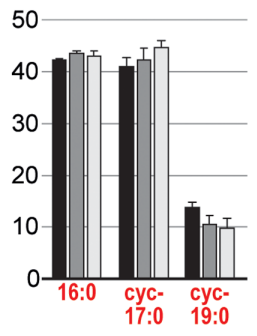

C

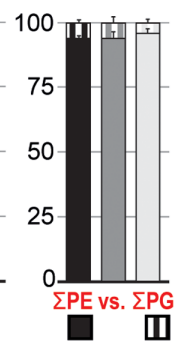

س 


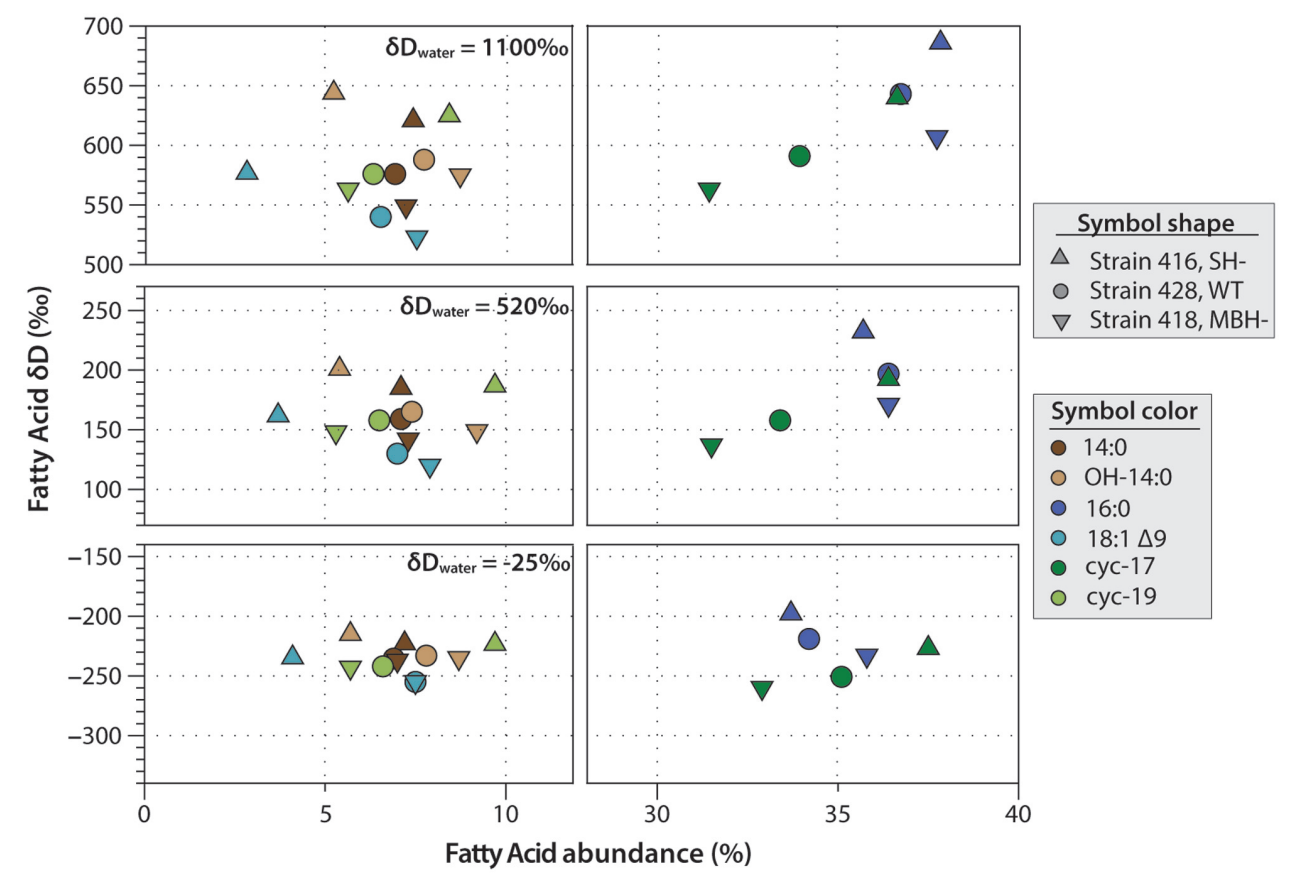

FIGURE 3 | Relative abundance and $\delta D_{F A}$ values of the six major fatty acids from $C$. necator cultures. The $\delta D_{\text {water }}$ values of culture medium are shown for each group. Error bars $( \pm 1 \mathrm{SD})$ are approximately the size of each symbol, or smaller.

curves for different fatty acids from the same strain are shifted along a nearly vertical trajectory. Such shifts are representative of primarily a change in net lipid/ $\mathrm{H}_{2}$ fractionation (see discussion in Zhang et al., 2009a), although differences between fatty acids are presumably a manifestation of differences in biosynthetic fractionation rather than differences in metabolic reactions of $\mathrm{H}_{2}$ per se. Zhang et al. (2009a) suggested that for heterotrophs the two fractionations (lipid/water and lipid/substrate) should likely be within $\sim 300 \%$ of each other, a region defined by the gray shading in Figure 5. In C. necator, the fractionation of $\mathrm{H}_{2}$ may be much more significant than that of water, leading to even lower $\alpha_{\mathrm{FA} / \mathrm{H} 2}$ to $\alpha_{\mathrm{FA} / \text { water }}$ ratios (above and to the left of the gray region). In either case, this analysis indicates that $90 \%$ or more of the fatty acid hydrogen was derived from water, not $\mathrm{H}_{2}$. The net fractionation between fatty acids and water can also be compared to those of $D$. autotrophicum grown chemoautotrophically; it derives lipid hydrogen exclusively from water, which allowed Campbell et al. (2009) to measure $\alpha_{\mathrm{FA} / \text { water }}$ directly (tan shaded zone in Figure 5). The fractionations for C. necator are near the upper end of this range.

A significant complication for this analysis regards the fact that the $\delta \mathrm{D}$ values of headspace $\mathrm{H}_{2}$ changed throughout the growth cultures, in a convoluted fashion (because of daily $\mathrm{H}_{2}$ additions and continuous equilibration with $\mathrm{H}_{2} \mathrm{O}$ ). The curves in Figure 5 were generated assuming $\delta \mathrm{D}_{\mathrm{H} 2}=-400 \%$ for all cultures, a value that is at the low end of the averages observed for any single culture (averages ranged from -267 to $-406 \%$; the generation of fractionation curves implicitly assumes a constant value of $R_{\mathrm{H} 2}$ for all cultures). Regardless, the shapes and relative positions of those curves do not depend on the assumed value. The assumed value for $\delta \mathrm{D}_{\mathrm{H} 2}$ does affect the absolute vertical position (i.e., the implied lipid/ $\mathrm{H}_{2}$ fractionation factor) of the curves, with a less negative $\delta \mathrm{D}_{\mathrm{H} 2}$ value moving the curves downward. But since all fatty acids derive from the same $\mathrm{H}_{2}$ source, all of the curves move up and down together as $\delta D_{\mathrm{H} 2}$ is changed. For our analysis here, the most conservative assumption would be $\delta \mathrm{D}_{\mathrm{H} 2}=-632 \%$, i.e., the most negative value measured at any time point in any one of the cultures. Under that assumption, the upper bound of the gray shaded region in Figure 5 would intersect the fractionation curve at $X_{\text {water }}=0.92$, instead of the $X_{\text {water }}=0.95$ obtained by assuming $\delta D_{\mathrm{H} 2}=-400 \%$. Assuming higher (less negative) $\delta \mathrm{D}_{\mathrm{H} 2}$ values would only result in even higher inferred $X_{\text {water }}$ values. The conclusion that little to no lipid hydrogen derived from $\mathrm{H}_{2}$ is thus robust, regardless of the actual, growth-integrated $\delta \mathrm{D}$ value of headspace $\mathrm{H}_{2}$.

\section{DISCUSSION}

A first-order observation for C. necator grown on $\mathrm{H}_{2}+\mathrm{O}_{2}$ is that net $\mathrm{D} / \mathrm{H}$ fractionations of its fatty acids relative to growth medium water fall in the range of $-199 \%$ to $-236 \%$, indistinguishable from those of many photoautotrophic algae and bacteria (Sessions et al., 1999; Chikaraishi et al., 2004; Zhang and Sachs, 2007; Zhang et al., 2009b; Chivall et al., 2014; Heinzelmann et al., 2015a). Deleting either the membrane-bound hydrogenase $(\mathrm{MBH})$ enzyme or the soluble hydrogenase $(\mathrm{SH})$ enzyme has only a small effect on $\delta \mathrm{D}_{\mathrm{FA}}$ values, changing them by $25 \%$ or less (Figure 3 and Table 3 ). Fractionation curves, 
TABLE 3 | Fatty acid $\delta$ D values from $C$. necator cultures.

\begin{tabular}{|c|c|c|c|}
\hline C. necator strain: & Wild type & $\mathbf{S H}^{-}$ & $\mathrm{MBH}^{-}$ \\
\hline \multicolumn{4}{|l|}{ Fatty acid } \\
\hline \multicolumn{4}{|l|}{$\delta D_{\text {water }} \approx-25 \% 0^{a}$} \\
\hline $14: 0$ & $-235.5(0.5)^{b}$ & $-222.8(0.9)$ & $-237(3)$ \\
\hline $3-\mathrm{OH} 14: 0$ & $-233(2)$ & $-215(2)$ & $-235(3)$ \\
\hline $16: 0$ & $-219(2)$ & $-197.7(0.4)$ & $-233(3)$ \\
\hline сус-17:0 & $-250.8(0.5)$ & $-226.7(0.4)$ & $-260(1)$ \\
\hline $18: 1 \Delta 9$ & $-255(1)$ & $-234.6(0.6)$ & $-255(2)$ \\
\hline сус-19:0 & $-242(4)$ & $-223.2(0.6)$ & $-243(6)$ \\
\hline Weighted mean ${ }^{c}$ & -237 & -216 & -245 \\
\hline \multicolumn{4}{|l|}{$\delta D_{\text {water }} \approx 520 \%$} \\
\hline $14: 0$ & $159(3)$ & $185(1)$ & $142(1)$ \\
\hline 3-OH14:0 & $165.1(0.8)$ & $201(1)$ & $149(3)$ \\
\hline $16: 0$ & $197.0(0.8)$ & $232.3(0.7)$ & $171(5)$ \\
\hline сус-17:0 & $157.9(0.7)$ & $192.5(0.8)$ & $137(3)$ \\
\hline $18: 1 \Delta 9$ & $130.0(0.9)$ & $162(1)$ & $120(9)$ \\
\hline сус-19:0 & $157.9(0.7)$ & $187(2)$ & $148(6)$ \\
\hline Weighted mean & 172 & 205 & 150 \\
\hline \multicolumn{4}{|l|}{$\delta D_{\text {water }} \approx 1100 \%$} \\
\hline $14: 0$ & 576 & $621(3)$ & $549(5)$ \\
\hline 3-OH14:0 & 588 & $644(9)$ & $575(1)$ \\
\hline $16: 0$ & 643 & $686(2)$ & $607(3)$ \\
\hline сус- $17: 0$ & 591 & $640(2)$ & $563(2)$ \\
\hline $18: 1 \Delta 9$ & 540 & $577(4)$ & $523(1)$ \\
\hline сус-19:0 & 576 & $625(2)$ & $563(1)$ \\
\hline Weighted mean & 605 & 653 & 576 \\
\hline
\end{tabular}

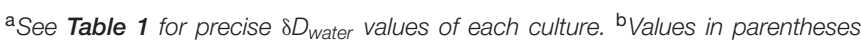
are one standard deviation of replicate analyses of the same sample; missing value indicates a single analysis of that sample. 'Mean weighted by relative abundance of each fatty acid.

constrained by experiments in which the $\delta \mathrm{D}$ of growth water was varied systematically, are also consistent with very little lipid hydrogen (approximately $10 \%$ or less) being derived from $\mathrm{H}_{2}$, and with lipid/water fractionations in the neighborhood of $200 \%$ (Figure 5). All lines of experimental evidence therefore point to a minimal contribution of $\mathrm{H}_{2}$ metabolism to lipid $\delta \mathrm{D}$ values in C. necator.

There are, nevertheless, subtle but systematic differences in $\delta \mathrm{D}_{\mathrm{FA}}$ values between the wild type and hydrogenase mutants. In our main experiment, fatty acids from $\mathrm{SH}^{-}$were $19.2 \%$ more D-enriched than those from the wild type (range 18$24 \% 0$ ) in experiments with $\delta \mathrm{D}_{\text {water }}=-25 \%$. Differences between cultures grown on more $\mathrm{D}$-enriched water were in the same directions, but even larger (up to $46 \% 0$ in magnitude). In comparing fatty acids from $\mathrm{MBH}^{-}$and wild-type strains, there was no significant difference in cultures with $\delta D_{\text {water }}=-25 \%$, but significant differences in more D-enriched cultures. $\mathrm{MBH}^{-}$ fatty acids were D-depleted relative to the wild type in those experiments. Such differences cannot plausibly be attributed to changes in fatty acid abundance, as there were barely detectable variations in their relative abundance between the strains. Schouten et al. (2006) and Leavitt et al. (2016) have previously suggested growth rate as an important influence on fractionation, with faster growing strains exhibiting larger

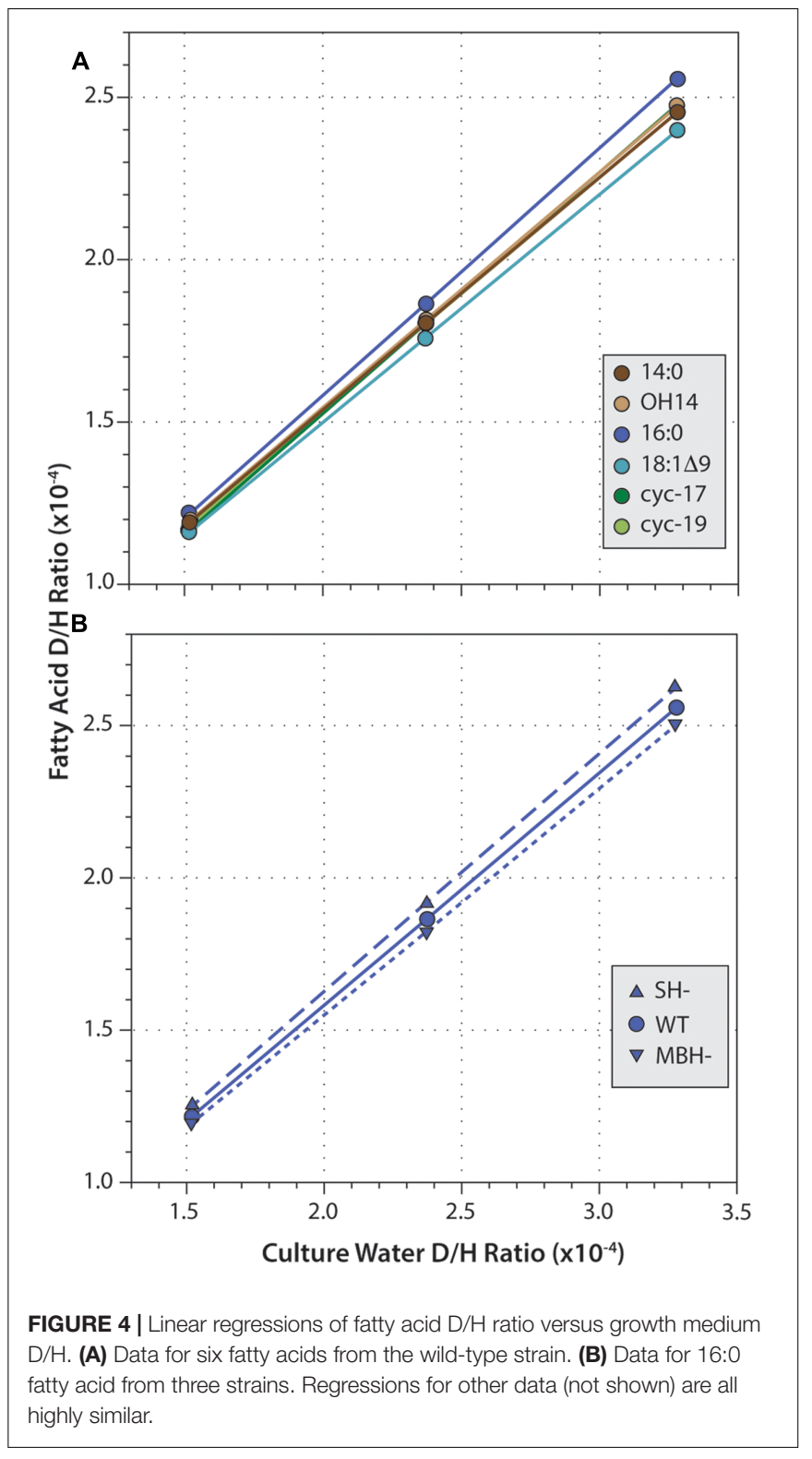

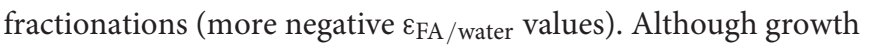
rates could not be quantified in our experiments (see section "Culture Conditions and Growth"), the relationship between growth rates and fractionations was qualitatively similar to that observed by Schouten et al. (2006) and Leavitt et al. (2016). Notably, however, the size of the effect in the latter study was $\sim 100 \%$, whereas in our data it is only $25 \%$ or less. Somewhat contradictorily, Osburn et al. (2016) have shown for a large survey of anaerobic heterotrophs that, in general, fractionation is not correlated with growth rate, although there is possibly a weak correlation amongst the slowest-growing organisms. The influence of growth rate on $\mathrm{D} / \mathrm{H}$ fractionations in Cupriavidus thus remains uncertain.

Below, we address three remaining questions. First, what role (if any) do hydrogenase enzymes play in setting the isotopic composition of lipids, either in this organism or 


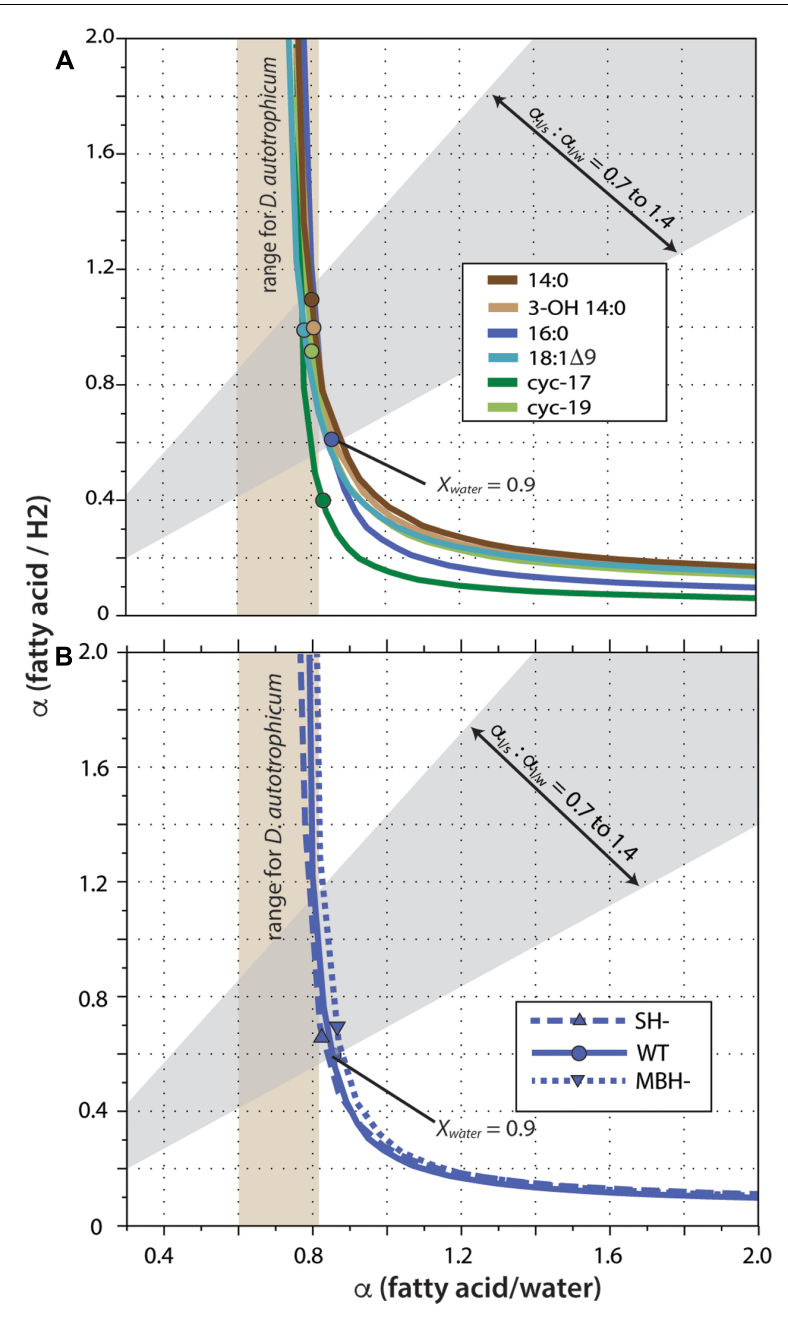

FIGURE 5 | Fractionation curves for different fatty acids from the wild-type strain (A), or for 16:0 fatty acid from different strains (B). Each curve represents the set of all possible combinations of lipid/ $/ \mathrm{H}_{2}$ fractionation, lipid/water fractionation, and $X_{\text {water }}$ (fraction of lipid hydrogen derived from water) that fit a given set of experimental constraints. Circles mark the point on each curve corresponding to the value $X_{\text {water }}=0.90$; these are marked only to facilitate comparison between curves, and do not otherwise have particular meaning. The gray shaded region outlines values where the two fractionations differ by no more than $300 \%$, as proposed by Zhang et al. (2009a). Tan shaded region indicates lipid/water fractionations measured for D. autotrophicum that uses only water hydrogen (Campbell et al., 2009).

other hydrogenotrophs? Second, are strong D-depletions truly a hallmark of chemoautotrophic metabolism, as has been previously proposed (Zhang et al., 2009a)? Third, is it coincidence that the $\delta \mathrm{D}_{\mathrm{FA}}$ values of $C$. necator are indistinguishable from those of plants?

\section{Hydrogenase Influence on Fatty Acid Isotopic Compositions}

In prokaryotes, including C. necator, n-alkyl fatty acids are synthesized in the cytoplasm from the precursor acetyl-CoA, which is a key intermediate in many pathways (Heath et al.,
2002). During the elongation cycle, hydrogen is transferred to fatty acids from NADPH and - if certain alternative enzymes are present - from NADH (Campbell and Cronan, 2001) during two separate reduction steps. The first, catalyzed by the FabG subunit, reduces 3-ketoacyl-ACP to 3-hydroxyaclACP. In most studied organisms this subunit is known to use only NADPH as cofactor. However, the genome of C. necator H16 contains at least one FabG with 97\% amino acid similarity to a FabG from Cupriavidus taiwanensis that uses NADH as cofactor (Javidpour et al., 2014). The second reduction, of enoyl-ACP to 3-hydroxyenoyl-ACP, is catalyzed by the FabI subunit, which is well known to use both $\mathrm{NADH}$ and NADPH cofactors. Thus, it appears likely that C. necator has the capability of using $\mathrm{NADH}$, probably in combination with NADPH, as a hydride donor for fatty acid biosynthesis.

The immediate sources of hydrogen to fatty acid biosynthesis are thus acetyl-CoA, $\mathrm{NAD}(\mathrm{P}) \mathrm{H}$, and water (for further discussion, see Osburn et al., 2016). The activities of precursor pathways during autotrophic growth are not known, but in C. necator cultivated on gluconate, the major source of NADPH and acetyl-CoA is the oxidation of sugars via the pentose phosphate (PP) pathway (Lee et al., 2003). In C. necator, carbohydrate precursors (which flow to $\mathrm{PP}$ ) are produced by the Calvin-Benson-Bassham (CBB) cycle (Bowien and Kusian, 2002), which in turn uses NADH as the reductant. NADH is itself generated largely from $\mathrm{H}_{2}$ via the $\mathrm{SH}$ (see below).

C. necator $\mathrm{H} 16$ reportedly possesses four putative hydrogenases, each assigned to a different group within the [NiFe] hydrogenase class (Schwartz et al., 2003; Vignais and Colbeau, 2004). The regulatory hydrogenase (RH) belongs to Group 2, the cytoplasmic $\mathrm{H}_{2}$ sensors. $\mathrm{RH}$ regulates biosynthesis of the two energy-generating hydrogenases and is not directly involved in metabolism (Lenz and Friedrich, 1998). MBH belongs to Group 1, the uptake hydrogenases. The $\mathrm{SH}$ belongs to Group 3, the bidirectional, heteromultimeric, cytoplasmic hydrogenases. $\mathrm{MBH}$ and $\mathrm{SH}$ both generate energy by the heterolytic cleavage of $\mathrm{H}_{2}$. In [NiFe] hydrogenases, the process consists of two steps: first $\mathrm{H}_{2}$ is cleaved into $\mathrm{H}^{-}$(hydride) and $\mathrm{H}^{+}$ions, and then the two electrons are extracted from the hydride leaving a second $\mathrm{H}^{+}$(de Lacey et al., 2005). The electrons are transferred to an acceptor, while the protons (or deuterons) are transported out of the protein to the surrounding water (Vignais, 2005). Despite these shared properties, $\mathrm{MBH}$ and SH differ in both location and function, and they probably have different effects on the pools of water and NADH available for biosynthesis. The reaction network involving hydrogenases and these intermediates must be considered as a whole (Figure 6). A fourth $[\mathrm{NiFe}]$ hydrogenase $(\mathrm{Hyd} 4)$ is apparently coded for on the megaplasmid of C. necator H16 (Schwartz et al., 2003). However, since the triple mutant lacking $\mathrm{RH}, \mathrm{MBH}$, and $\mathrm{SH}$ shows no detectable hydrogenase activity, the function of the Hyd4 genes is unknown and is not considered further here.

$\mathrm{MBH}$ catalyzes the unidirectional oxidation of $\mathrm{H}_{2}$, which is ultimately coupled to $\mathrm{O}_{2}$ reduction via a respiratory chain (Schink and Schlegel, 1979). Located in the periplasmic space, $\mathrm{MBH}$ is anchored to the cytoplasmic membrane by a $b$-type 


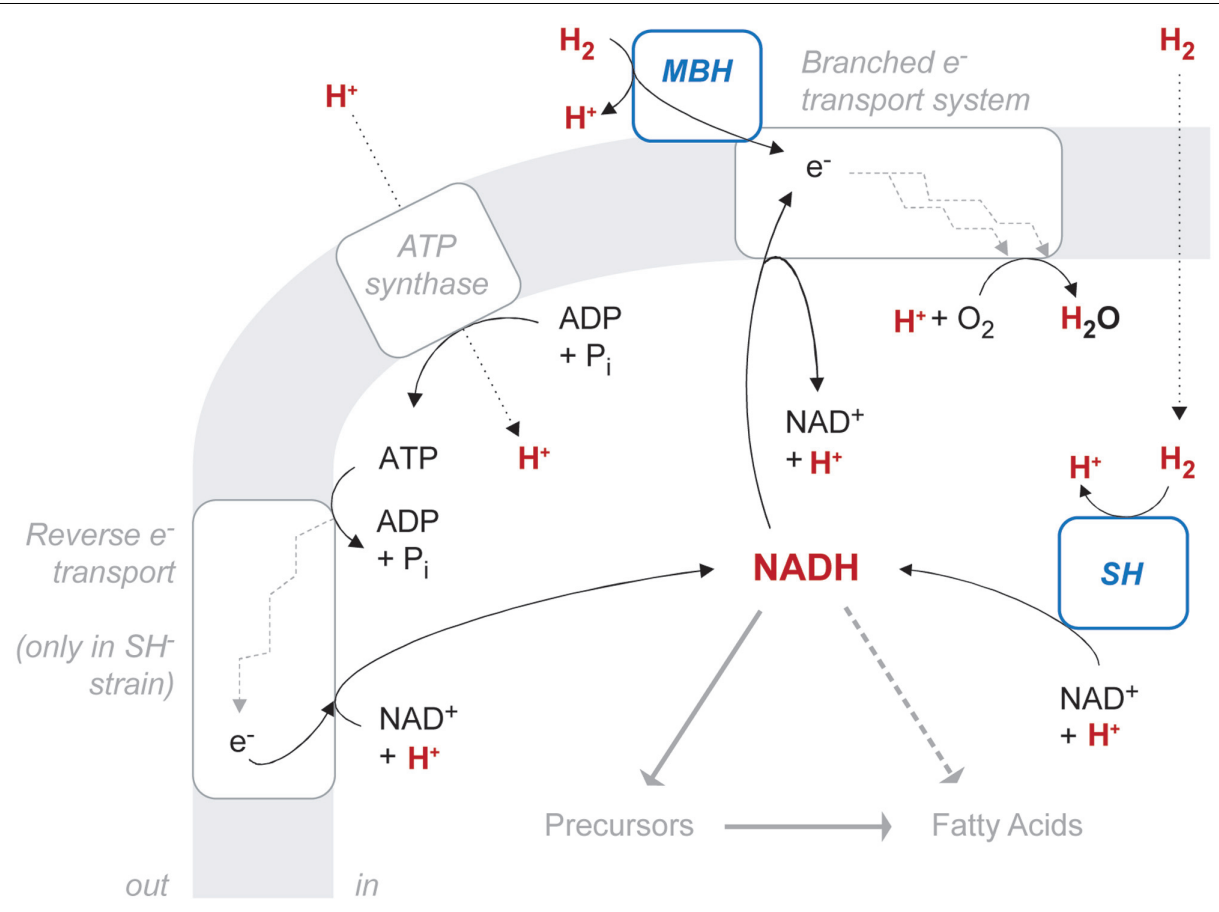

FIGURE 6 | The configuration of hydrogenase enzymes and hydrogen pathways in C. necator. The gray shaded area represents the cytoplasmic membrane. ATP synthase and the electron transport system are associated with the membrane, as is the membrane-bound hydrogenase (MBH), which is located in the periplasmic space. The soluble hydrogenase (SH) is located in the cytoplasm. Hydrogen-bearing species are in red. Fine black arrows indicate chemical reactions or half-reactions (not stoichiometrically balanced). Dotted black arrows denote diffusion or transport across the membrane. Heavy gray arrows denote hydrogen flow from NADH to fatty acids, either through biosynthetic precursors (such as NADPH or acetate) or possibly by direct contribution (dashed).

cytochrome subunit, which is also the immediate acceptor of electrons from $\mathrm{H}_{2}$ oxidation (Friedrich and Schwartz, 1993; Bernhard et al., 1997). Electrons from $\mathrm{H}_{2}$ flow through the $b$-type cytochrome into the UQ pool, which feeds into the electron transport system. Electron transport complexes link the movement of electrons to the uptake of protons (generating ubiquinol) at the cytoplasmic side of the membrane and the release of protons at the periplasmic side (Komen et al., 1996). The resulting proton gradient supports ATP synthesis.

$\mathrm{SH}$ located in the cytoplasm, couples the oxidation of $\mathrm{H}_{2}$ to the reduction of $\mathrm{NAD}^{+}$(Schneider and Schlegel, 1976). Reduction is thought to occur via electron transfer through $\mathrm{Fe}-$ $S$ clusters of the hoxU subunit, rather than as an intact hydride transfer (Burgdorf et al., 2006). The enzyme is reversible, but $\mathrm{H}_{2}$ evolution from $\mathrm{NADH}$, in the presence of sodium dithionite, occurs at only $2.4 \%$ of the rate of $\mathrm{H}_{2}$ uptake with $\mathrm{NAD}^{+}$as electron acceptor (Schneider and Schlegel, 1976). This property is consistent with the demand for large amounts of NADH by the CBB cycle during autotrophic growth (Bowien and Kusian, 2002). Cells lacking SH can grow autotrophically, but only at a diminished rate, as observed in our study. Impaired growth may be due to inefficient production of NADH via ATP-dependent reverse electron transport (Komen et al., 1992). In our study, the $\mathrm{SH}^{-}$mutant exhibited an increase in relative abundance of UQ over both the wild type and $\mathrm{MBH}^{-}$strains (Figure 2B). This observation would be consistent with the upregulation of UQ in $\mathrm{SH}^{-}$cells to stimulate the rate of electron transport in the membrane.

The electron transport system of $C$. necator is central to the relationship of $\mathrm{SH}, \mathrm{MBH}$, and the $\mathrm{NADH}$ pool. $\mathrm{H}_{2}$ oxidation via $\mathrm{MBH}$ is not the sole source of electrons for transport. Instead, the system is branched, linking the oxidation of $\mathrm{H}_{2}$, $\mathrm{NADH}$, and succinate, through the UQ pool, to four different membrane-bound terminal oxidases (Komen et al., 1991a,b). The two major branches during exponential growth are a botype oxidase and a cytochrome $c$-containing pathway (which is itself branched, having two terminal oxidases). The fourth terminal oxidase, a $d$-type cytochrome, is active mainly during late exponential growth. In membranes from exponential-phase, autotrophic $C$. necator, electron flow from $\mathrm{H}_{2}$ via the bo-type oxidase accounts for $80 \%$ of ATP synthesis, while the cytochrome $c$-containing pathway plays "a minor role in energy conservation" (Komen et al., 1996). In a study comparing $\mathrm{SH}^{-}$and $\mathrm{MBH}^{-}$ mutants (different strains than the mutants in this study) to the wild type, Komen et al. (1992) found that the preferred pathway of electron transport is different for each strain, and the wild type reportedly favors the cytochrome $c$-containing pathway. That study also reported NADH dehydrogenase activity for each strain: $\mathrm{MBH}^{-}$cells exhibited $167 \%$ activity relative to wild-type cells, while $\mathrm{SH}^{-}$cells exhibited $30 \%$ activity relative to wild-type cells. We speculate that the relative activities of $\mathrm{NADH}$ dehydrogenase could be a factor linking growth rates 
to hydrogen-isotopic fractionations (as shown in Leavitt et al., 2016).

The preceding discussion makes it clear that there is no direct enzymatic link between the isotopic composition of $\mathrm{H}_{2}$ and lipids. Both uptake hydrogenases transfer only electrons, not hydrogen nuclei. The fact that $\mathrm{SH}^{-}$mutants are only slightly different in $\delta \mathrm{D}_{\mathrm{FA}}$ is consistent with this conclusion. Rather, the modest shifts in $\delta \mathrm{D}_{\mathrm{FA}}$ between the wild type, $\mathrm{SH}^{-}$, and $\mathrm{MBH}^{-}$ are likely due to one or more indirect effects. Several such effects can be considered, including: generation of metabolic water from $\mathrm{H}_{2}$, hydrogen-isotopic exchange between water and $\mathrm{H}_{2}$, generation of reducing equivalents via reverse electron transport, differing transhydrogenase activity and/or growth rate, and different partitioning of reducing power between catabolism and anabolism.

The different locations of SH and MBH provide two possible mechanisms of influence on the intracellular water pool: first the oxidation of $\mathrm{H}_{2}$ to $\mathrm{H}_{2} \mathrm{O}$, and second the catalyzed isotopic equilibration of $\mathrm{H}_{2}$ with $\mathrm{H}_{2} \mathrm{O}$ (i.e., independent of oxidation). $\mathrm{MBH}$, anchored to the outer surface of the cytoplasmic membrane, oxidizes $\mathrm{H}_{2}$ in the periplasmic space where the isotopic composition of that signal is presumably quickly mixed with extracellular water and therefore lost. In contrast, $\mathrm{SH}$ oxidizes $\mathrm{H}_{2}$ in the cytoplasm, where released $\mathrm{H}^{+}$will rapidly equilibrate with water and could thereby be incorporated into fatty acids. We thus can predict that hydrogenase influences on cellular water should be most pronounced in $\mathrm{MBH}^{-}$, which relies completely on $\mathrm{SH}$, and should be least pronounced in $\mathrm{SH}^{-}$.

Supplied $\mathrm{H}_{2}$ in our cultures was always strongly D-depleted relative to growth water, so catabolic oxidation of $\mathrm{H}_{2}$ to $\mathrm{H}_{2} \mathrm{O}$ should serve to lower water and thus fatty acid $\delta \mathrm{D}$ values. Assuming that this effect is strongest in the $\mathrm{MBH}^{-}$strain (as argued above), the predicted variation in $\delta \mathrm{D}_{\mathrm{FA}}$ would be $\mathrm{MBH}^{-}$ $<$ wild type $<\mathrm{SH}^{-}$, consistent with the observed variation. Because the isotopic contrast between $\mathrm{H}_{2}$ and $\mathrm{H}_{2} \mathrm{O}$ was greatest in those experiments with $\mathrm{D}$-enriched medium, this mechanism could also potentially explain the increasing differences between strains with increasing $\delta D_{\text {water. }}$.

The second process to consider is hydrogenase-catalyzed isotopic exchange between $\mathrm{H}_{2}$ and $\mathrm{H}_{2} \mathrm{O}$; the existence of this process is clearly indicated by the decrease in $\delta \mathrm{D}_{\mathrm{H} 2}$ over the course of the experiment. This decrease in $\delta \mathrm{D}_{\mathrm{H} 2}$ would tend to cause a corresponding enrichment in $\delta D_{\text {water }}$. Indeed, such enrichment was observed directly in the low- $\delta \mathrm{D}_{\text {water }}$ treatments, which were farthest from equilibrium with $\mathrm{H}_{2}$. Assuming that this effect is strongest in the $\mathrm{MBH}^{-}$strain, the predicted variation in $\delta \mathrm{D}_{\mathrm{FA}}$ would be $\mathrm{SH}^{-}<$wild type $<\mathrm{MBH}^{-}$. In fact, the opposite order was observed in this study. Therefore, the oxidation of $\mathrm{H}_{2}$ to $\mathrm{H}_{2} \mathrm{O}$ is the only mechanism of hydrogenase influence via the intracellular water pool that could yield the observed variation in $\delta \mathrm{D}_{\mathrm{FA}}$ between strains. A similar mechanism has previously been observed in E. coli (Kreuzer-Martin et al., 2006); however, the metabolic rates that generated a measurable effect in those experiments were likely orders of magnitude faster than those in our experiments. Growth rates in $C$. necator, at least as realized in our $\mathrm{O}_{2}$-limited batch cultures, may therefore be too slow to support any significant role for metabolic water.
Another obvious difference between the three C. necator strains is the necessity for the $\mathrm{SH}^{-}$mutant to employ reverse electron transport to generate $\mathrm{NADH}$ for carbon fixation (Figure 6). If this enzymatic reduction expresses a different kinetic isotope effect (KIE) than does $\mathrm{SH}$, then the differing isotopic composition of NADH could be transmitted into carbon fixed by the CBB cycle and ultimately into fatty acids, regardless of whether NADH or NADPH is specifically used by fatty acid synthase. Although this mechanism may contribute partly to the larger difference in $\delta \mathrm{D}_{\mathrm{FA}}$ between the $\mathrm{SH}^{-}$mutant and the wild type, it cannot play a role in the difference between the $\mathrm{MBH}^{-}$ mutant and the wild type, both of which are thought to reduce $\mathrm{NADH}$ via the $\mathrm{SH}$.

The genome of $C$. necator $\mathrm{H} 16$ contains at least four putative transhydrogenases, capable of interconverting NADH and NADPH. Given that such enzymes commonly express large KIEs (Jackson et al., 1999), the metabolic balance between NADH and NADPH production has the capacity to significantly affect the $\delta \mathrm{D}$ values of both cofactor pools, and thus fatty acids. We predict that the $\mathrm{MBH}^{-}$mutant would overproduce NADH (because of over-reliance on the $\mathrm{NAD}^{+}$-reducing $\mathrm{SH}$ ), whereas the $\mathrm{SH}^{-}$mutant is demonstrably underproducing $\mathrm{NADH}$. Isotope effects of the specific $C$. necator transhydrogenases have not been reported, but assuming they are similar to most other studied examples, i.e., normal KIEs, overproduction of NADH should emphasize NADH $\rightarrow \mathrm{NADPH}$ and thus D-depletion of $\mathrm{NADPH}$, whereas underproduction should emphasize NADH $\leftarrow \mathrm{NADPH}$ and D-enrichment of NADPH. If NADPH is the primary cofactor used for fatty acid synthesis, the predicted order of D-enrichment would be $\mathrm{MBH}^{-}<$wild type $<\mathrm{SH}^{-}$, which matches the observed data; if NADH were the primary cofactor, the predicted order would be reversed.

Finally, if NADH is used for biosynthesis, it is possible that the competition between anabolic and catabolic demands leads to shifts in isotopic composition. In this model, the NADH pool represents a branch point in the flow of hydrogen from water to lipids. While the hydrogen flux from NADH to lipids is assumed to be similar in all strains (similar FA abundances), there is evidence that the flux from $\mathrm{NADH}$ to water (i.e., respiration) varies systematically among strains. This flux represents the oxidation of NADH for electron transport, which is catalyzed by $\mathrm{NADH}$ dehydrogenase. As noted above, NADH dehydrogenase activity varies among strains in the order $\mathrm{SH}^{-}<$wild type $<$ $\mathrm{MBH}^{-}$(Komen et al., 1992). If the isotope effects associated with catabolic versus anabolic utilization of NADH differ, which seems plausible, then changes in the branching ratio between these two fates will lead to changes in the isotopic compositions, even with constant NADH supply (see Hayes, 2001). We cannot predict the direction of such effects without knowing the specific isotope effects associated with the two pathways, and either direction is plausible.

In summary, there are several possible mechanisms by which loss of $\mathrm{SH}$ or $\mathrm{MBH}$ could lead to modest shifts in lipid $\delta \mathrm{D}$; none of them involve direct transfer of hydrogen isotopes from $\mathrm{H}_{2}$ to $\mathrm{NAD}(\mathrm{P}) \mathrm{H}$. Oxidation of $\mathrm{H}_{2}$ to cellular water is possible but, given the slow growth in our experiments, is perhaps unlikely to be significant. Changes in the interconversion of 


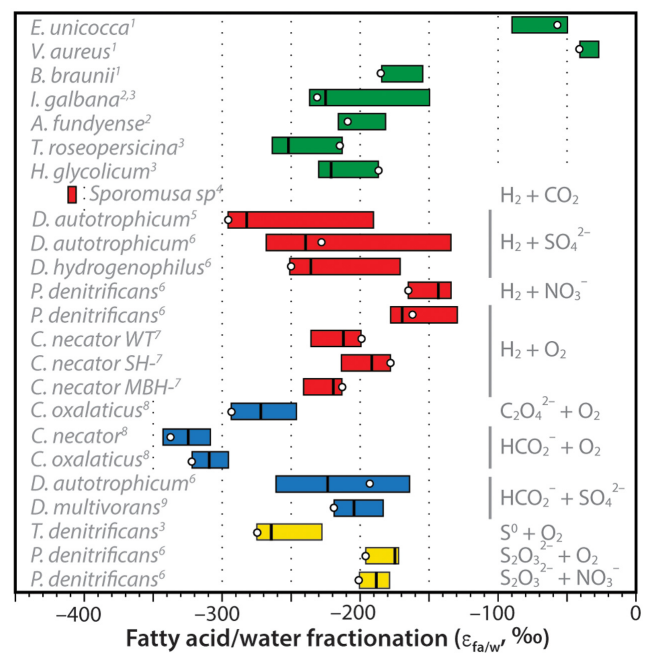

FIGURE 7 | Compilation of measured hydrogen-isotopic fractionations between fatty acids and growth water (expressed as

$\varepsilon_{\mathrm{FA}} /$ water $=\left[\left(\delta \mathrm{D}_{\mathrm{FA}}+1\right) /\left(\delta \mathrm{D}_{\text {water }}+1\right)\right]$. For each organism, colored bar represents the range of values for all measured fatty acids in that organism, vertical line is the weighted-mean value, circle is the value for palmitic acid (which is often the most abundant FA). Bars are colored coded for photoautotrophs (green), $\mathrm{H}_{2}$-using chemoautotrophs (red), formate- or oxalate-using chemoautotrophs (blue), or sulfur-oxidizing chemoautotrophs (yellow). Specific electron donors and acceptors are noted at right, species are noted at left. Data sources: ${ }^{1}$ Zhang and Sachs (2007); ${ }^{2}$ Sessions et al. (1999); ${ }^{3}$ Heinzelmann et al. (2015b); ${ }^{4}$ Valentine et al. (2004); ${ }^{5}$ Campbell et al. (2009); ${ }^{6}$ Osburn et al. (2016); ${ }^{7}$ This study; ${ }^{8}$ Zhang et al. (2009a); ${ }^{9}$ Dawson et al. (2015).

$\mathrm{NADH}$ and NADPH, or in the downstream fates of NADH, are plausible. Because hydrogenase enzymes are intimately linked to conservation of cellular reducing equivalents, and thus the NADPH pool, similar linkages are likely in most other organisms that have hydrogenase enzymes.

\section{Comparison with Other Organisms}

The net $\mathrm{D} / \mathrm{H}$ fractionations between fatty acids and water exhibited by $C$. necator can be compared to other organisms growing autotrophically (Figure 7 ). These include the marine, unicellular algae Isochrysis galbana and Alexandrium fundyense; the freshwater green algae Eudorina unicocca, Volvox aureus, and Botryococcus braunii; the photosynthetic purple sulfur bacteria Thiocapsa roseopersicina and Halochromatium glycolicum; $\mathrm{H}_{2}$-consuming cultures of the acetogenic bacterium Sporomusa sp. (DMG 58), the sulfate-reducing bacteria Desulfobacterium autotrophicum and Desulfobacter hydrogenophilus, and nitratereducing bacterium Paracoccus denitrificans; formate- and oxalate-consuming cultures of $C$. necator, $C$. oxalaticus, D. autotrophicum, and Desulfovibrio multivorans that are effectively chemoautotrophic with respect to hydrogen sources; and sulfur-oxidizing cultures of the bacteria Thiobacillus denitrificans and $P$. denitrificans. Data sources are provided in the caption of Figure 7.

Zhang et al. (2009a) had originally hypothesized that substantial D-depletion of lipids relative to growth water was a potential hallmark of chemoautotrophic organisms. This hypothesis was influenced heavily by the early results of Valentine et al. (2004) for Sporomusa sp. and Zhang et al. (2009a) for C. necator and C. oxalaticus, all of which were more D-depleted than photoautotrophs. A precise mechanistic explanation for the D-depletion was not offered. Additional data on autotrophic fractionations (Figure 7) are now fairly clear in disproving this hypothesis: $\mathrm{D} / \mathrm{H}$ fractionations in chemoautotrophs are not significantly different, on average, from those in unicellular photoautotrophs. Fractionations are still larger than those for most terrestrial plants (e.g., Sachse et al., 2012), but this is a consequence of soil and leaf water evaporation rather than a biochemical effect. Some heterotrophs also exhibit lipid/water fractionations that are in a similar range (e.g., Osburn et al., 2016), although many are more D-enriched for reasons that are still not entirely understood.

Although the distributions of chemoautotrophic and photoautotrophic fractionations are largely overlapping, there are several examples of chemoautotrophs that lie well outside the known range for photoautotrophs. These include D. autotrophicum grown on $\mathrm{H}_{2}$, and C. necator and C. oxalaticus grown on formate; but most of all, Sporomusa emerges as a clear anomaly. With this being the only studied example of a Firmicute or of an acetogenic organism, we do not speculate as to the uniqueness or biochemical basis of its anomalous D-depletion. Regardless, while it is clear that strong D-depletions are not ubiquitous amongst chemoautotrophs, it remains possible that abnormally D-depleted lipids could still be markers for certain metabolic pathways. This clearly requires further study, both to understand the phylogenetic distribution as well as the underlying mechanisms of the signal.

\section{Similarity of C. necator and Algal Fractionations}

A conspicuous feature of Figure 7 is the similarity of fractionations in C. necator grown on $\mathrm{H}_{2}$ and those in photoautotrophic bacteria and algae. Given that hydrogen metabolism in C. necator contributes little or no hydrogen to lipids, this leads us to question whether the similarity is merely a coincidence. Both groups of organisms use the same biosynthetic pathway for fatty acids, fueled by $\mathrm{NAD}(\mathrm{P}) \mathrm{H}$. However, sources of the latter cofactor differ significantly between knallgas bacteria and photoautotrophs.

In oxygenic photosynthesizers, reducing equivalents are conserved mainly as NADPH during the light reactions of photosynthesis. The electron-transport chain of photosystem I ultimately passes a single low-potential electron to ferredoxin, an iron-sulfur protein. Since iron atoms carry the reducing equivalent, ferredoxin is an electron carrier, not a hydride carrier. Reduced ferredoxin then diffuses to the membrane-bound enzyme ferredoxin-NADP ${ }^{+}$reductase (FNR). The prosthetic group of FNR is flavin adenine dinucleotide (FAD), which can accumulate two electrons from (two) ferredoxins and two hydrogen nuclei $\left(\mathrm{H}^{+}\right.$or $\left.\mathrm{D}^{+}\right)$from solution, thereby being reduced to $\mathrm{FADH}_{2} \cdot \mathrm{FADH}_{2}$ will in turn transfer these reducing equivalents as a hydride $\left(\mathrm{H}^{-}\right)$ion to $\mathrm{NADP}^{+}$ 
with stereospecificity (Ammeraal et al., 1965). The catalytic mechanism of FNR is thought to involve a ternary complex of ferredoxin, $\mathrm{FAD}$, and $\mathrm{NADP}^{+}$, and electron/hydride transfer is both rapid and thermodynamically reversible. A large deuterium isotope effect $\left(k_{\mathrm{H}} / k_{D} \sim 6\right)$ in vitro is consistent with intact hydride transfer (Tejero et al., 2007). The overall direction of the reaction is expected to be pushed strongly in the forward direction under physiologic conditions of photosynthesis, possibly via reductive inhibition by $\mathrm{NADPH}$ (Carrillo and Ceccarelli, 2003).

In $C$. necator, reducing equivalents are conserved primarily as $\mathrm{NADH}$, which is generated from $\mathrm{NAD}^{+}$by the $\mathrm{SH}$. $\mathrm{SH}$ is a two-domain enzyme, with one module representing the heterodimeric[NiFe] hydrogenase encoded by hoxH and hoxY, and the other a flavin-containing NADH dehydrogenase module encoded by hoxF and hoxU (Burgdorf et al., 2006). The former module accomplishes the oxidation of $\mathrm{H}_{2}$, passing electrons (but not hydrogen atoms) to the latter to reduce $\mathrm{NAD}^{+}$to $\mathrm{NADH}$. The NADH dehydrogenase module is quite similar to the mitochondrial Complex I (NADH:UQ oxidoreductase), and uses flavin mononucleotide (FMN) as its prosthetic group. Electron transfer from the hydrogenase module to the redox module is accomplished via a series of $\mathrm{Fe}-\mathrm{S}$ clusters. The catalytic mechanism of SH has not been studied, but in Complex I (which operates in the opposite direction to $\mathrm{SH}$, oxidizing $\mathrm{NADH}$ and reducing $U Q$ ) it involves intact hydride transfer from $\mathrm{NADH}$ to FMN. The reaction of $\mathrm{SH}$ in the forward direction presumably accumulates two electrons and two protons on FMN, followed by hydride transfer from reduced $\mathrm{FMNH}_{2}$ to $\mathrm{NAD}^{+}$. Thus in C. necator, water - not $\mathrm{H}_{2}$ - would be the source of hydrogen to $\mathrm{FMNH}_{2}$ and $\mathrm{NADH}$.

In both photosynthesis and hydrogenase-based chemoautotrophy, then, $\mathrm{NAD}(\mathrm{P})^{+}$is reduced by electrons transferred from iron-sulfur carriers to a flavin prosthetic group, with a hydrogen nucleus $\left(\mathrm{H}^{+}\right.$or $\left.\mathrm{D}^{+}\right)$acquired from solution, and then by hydride transfer from the reduced flavin to the nucleotide in a fast catalytic step. We do not yet know whether the observed fractionations are the result of equilibrium or KIEs, either during reduction of the flavin ring or during hydride transfer to the nucleotide. Regardless, the fact that these two enzymes with similar prosthetic groups express similar fractionations is arguably not a coincidence. Given the apparent constancy of the fractionation across a wide variety of plants, photosynthetic algae and bacteria, and (now) chemoautotrophic bacteria, we suggest that it may represent a general feature of many enzymes that use flavins as redox carriers.

\section{CONCLUSION}

C. necator $\mathrm{H} 16$ growing aerobically on $\mathrm{H}_{2}+\mathrm{CO}_{2}$ exhibited net $\mathrm{D} / \mathrm{H}$ fractionations between fatty acids and water that range from $-199 \%$ to $-236 \%$ (average $-217 \%$ ), indistinguishable from those observed in most photoautotrophic algae and bacteria. Diverse lines of evidence, including examination of known biochemistry, study of hydrogenase mutants, and fractionation curves developed by modulating the isotopic composition of growth water, all indicate that $\mathrm{H}_{2}$ contributed little or no hydrogen to lipids. Instead, the observed fractionation must have resulted from the reduction of $\mathrm{NADH}$ with hydrogen nuclei from water, using only electrons supplied by $\mathrm{H}_{2}$. Small differences in fatty acid $\delta \mathrm{D}$ values $(\leq 25 \% 0)$ were observed between hydrogenase-deficient mutants and the wild type. These differences can likely be explained by changes in growth rate or efficiency, cellular localization of $\mathrm{H}_{2}$ oxidation, and/or competing demands for NADH. The lipid-water fractionations exhibited by $C$. necator are common, though not ubiquitous, among chemoautotrophs for which fractionations have been reported. The hypothesis that chemoautotrophic and photoautotrophic biomass can be distinguished by their $\delta \mathrm{D}$ values is therefore falsified, although at least some chemoautotrophs are significantly D-depleted for reasons not yet known. A fractionation of roughly $-200 \% 0$ appears to be common to many $\mathrm{NAD}(\mathrm{P})^{+}$reductases.

\section{AUTHOR CONTRIBUTIONS}

BC planned the experiments, conducted research, analyzed the data, and wrote the manuscript. AS performed lipid analyses, analyzed the data, and wrote the manuscript. DF helped with microbial cultures and gas isotope analyses. BP provided analysis of genomic data for strain H16. QQ cultivated and sampled microbial cultures. MK performed the polar lipid analysis, DV planned and supervised the experiments, analyzed the data, and edited the manuscript.

\section{FUNDING}

This work was supported by the NASA Graduate Student Researcher Program (to BC) and grants from the National Science Foundation (EAR-0311894 to DV and AS; OCE-1046144 to DV; EAR-1529120 to AS).

\section{ACKNOWLEDGMENTS}

The authors gratefully acknowledge Robert Petty, Chao Li, and Fenfang $\mathrm{Wu}$ for assistance with various isotopic analyses. We thank the three reviewers, who contributed substantively to improving the manuscript.

\section{SUPPLEMENTARY MATERIAL}

The Supplementary Material for this article can be found online at: http://journal.frontiersin.org/article/10.3389/fmicb. 2017.01886/full\#supplementary-material

FIGURE S1 | Bar charts of hydrogen-isotopic fractionations between fatty acids and growth water, grouped by fatty acid structure and color-coded by strain. Top, middle, and bottom plots are data from cultures with no D-enrichment, medium D-enrichment, and high D-enrichment, respectively.

TABLE S1 | Complete dataset of fatty acid structure, abundance, and isotopic composition; polar lipid structure and abundance; and bacterial growth measurements. 


\section{REFERENCES}

Ammeraal, R. N., Krakow, G., and Vennesland, B. (1965). The stereospecificity of the hill reaction with diphosphopyridine nucleotide. J. Biol. Chem. 240, 1824-1828.

Bernhard, M., Benelli, B., Hochkoeppler, A., Zannoni, D., and Friedrich B. (1997). Functional and structural role of the cytochrome $b$ subunit of the membranebound hydrogenase complex of Alcaligenes eutrophus H16. Eur. J. Biochem. 248, 179-186. doi: 10.1111/j.1432-1033.1997.00179.x

Bowien, B., and Kusian, B. (2002). Genetics and control of $\mathrm{CO}_{2}$ assimilation in the chemoautotroph Ralstonia eutropha. Arch. Microbiol. 178, 85-93. doi: 10.1007/ s00203-002-0441-3

Burgdorf, T., Lenz, O., Buhrke, T., van der Linden, E., Jones, A. K., Albracht, S. P. J., et al. (2006). [NiFe]-hydrogenases of Ralstonia eutropha H16: Modular enzymes for oxygen-tolerant biological hydrogen oxidation. J. Molec. Microbiol. Biotechnol. 10, 181-196. doi: 10.1159/000091564

Campbell, B. J., Li, C., Sessions, A. L., and Valentine, D. L. (2009). Hydrogen isotopic fractionation in lipid biosynthesis by $\mathrm{H}_{2}$-consuming Desulfobacterium autotrophicum. Geochim. Cosmochim. Acta 73, 2744-2757. doi: 10.1016/j.gca. 2009.02.034

Campbell, J. W., and Cronan, J. E. (2001). Bacterial fatty acid biosynthesis: targets for antibacterial drug discovery. Ann. Rev. Microbiol. 55, 305-332. doi: 10.1146/ annurev.micro.55.1.305

Carrillo, N., and Ceccarelli, E. A. (2003). Open questions in ferredoxin-NADP+ reductase catalytic mechanism. Eur. J. Biochem. 270, 1900-1915. doi: 10.1046/j. 1432-1033.2003.03566.x

Chikaraishi, Y., Naraoka, H., and Poulson, S. R. (2004). Hydrogen and carbon isotopic fractionations of lipid biosynthesis among terrestrial (C3, C4 and CAM) and aquatic plants. Phytochemistry 65, 1369-1381. doi: 10.1016/j. phytochem.2004.03.036

Chivall, D., M'Boule, D., Sinke-Schoen, D., Sinninghe Damsté, J. S., Schouten, S., and van der Meer, M. T. J. (2014). The effects of growth phase and salinity on the hydrogen isotopic composition of alkenones produced by coastal haptophyte algae. Geochim. Cosmochim. Acta 140, 381-390. doi: 10.1016/j.gca.2014.05.043

Christie, W. W. (2003). Lipid Analysis, Vol. 15, 3rd ed. Bridgewater: The Oily Press, 416.

Davis, D. H., Doudorof, M., Stanier, R. Y., and Mandel, M. (1969). Proposal to reject the genus Hydrogenomonas: taxonomic implications. Int. J. Syst. Bacteriol. 19, 375-390. doi: 10.1099/00207713-19-4-375

Dawson, K. S., Osburn, M. R., Sessions, A. L., and Orphan, V. J. (2015). Metabolic associations with archaea drive shifts in hydrogen isotope fractionation in sulfate-reducing bacterial lipids in cocultures and methane seeps. Geobiology 13, 462-477. doi: 10.1111/gbi.12140

de Lacey, A. L., Fernandez, V. M., and Rousset, M. (2005). Native and mutant nickel-iron hydrogenases: unravelling structure and function. Coord. Chem. Rev. 249, 1596-1608. doi: 10.1016/j.ccr.2005.03.009

Dirghangi, S. S., and Pagani, M. (2013). Hydrogen isotope fractionation during lipid biosynthesis by Haloarcula marismortui. Geochim. Cosmochim. Acta. 119, 381-390. doi: 10.1016/j.gca.2013.05.023

Friedrich, B., and Schwartz, E. (1993). Molecular biology of hydrogen utilization in aerobic chemolithotrophs. Annu. Rev. Microbiol. 47, 351-383. doi: 10.1146/ annurev.mi.47.100193.002031

Hagemann, R., Nief, G., and Roth, E. (1970). Absolute isotopic scale for deuterium analysis of natural waters. Absolute $\mathrm{D} / \mathrm{H}$ ratio for SMOW. Tellus 22, 712-715. doi: 10.1111/j.2153-3490.1970.tb00540.x

Hayes, J. M. (2001). "Fractionation of carbon and hydrogen isotopes in biosynthetic processes," in Reviews in Mineralogy and Geochemistry: Stable Isotope Geochemistry, Vol. 43, eds J. Valley and D.R. Cole. Washington, DC: Mineralogical Society of America. 225-277

Heath, R. J., Jackowski, S., and Rock, C. O. (2002). "Fatty acid and phospholipid metabolism in prokaryotes," in Biochemistry of Lipids, Lipoproteins, and Membranes, 4th Edn, eds D. E. Vance and J. E. Vance (New York, NY: Elsevier), 55-92.

Heinzelmann, S. M., Chivall, D., M'Boule, D., Sinke-Schoen, D., Villanueva, L., Sinninghe Damsté, J. S., et al. (2015a). Comparison of the effect of salinity on the $\mathrm{D} / \mathrm{H}$ ratio of fatty acids of heterotrophic and photoautotrophic microorganisms. FEMS Microbiol. Lett. 362:fnv065. doi: 10.1093/femsle/fnv065
Heinzelmann, S. M., Villanueva, L., Sinke-Schoen, D., Sinninghe Damsté, J. S., Schouten, S., and van der Meer, M. T. J. (2015b). Impact of metabolism and growth phase on the hydrogen isotopic composition of microbial fatty acids. Front. Microbiol. 6:408. doi: 10.3389/fmicb.2015.00408

Horibe, Y., and Craig, H. (1995). D/H fractionation in the system methanehydrogen-water. Geochim. Cosmochim. Acta 59, 5209-5217. doi: 10.1016/00167037(95)00391-6

Hungate, R. E. (1969). “A roll tube method for cultivation of strict anaerobes," in Methods in Microbiology, Vol. 3b eds J. R. Norris and D. W. Ribbons (London: Academic Press, Inc.), 117-132.

Jackson, J. B., Peake, S. J., and White, S. A. (1999). Structure and mechanism of proton-translocating transhydrogenase. FEBS Lett. 464, 1-8. doi: 10.1016/ S0014-5793(99)01644-0

Javidpour, P., Pereira, J. H., Goh, E.-B., McAndrew, R. P., Ma, S. M., Friedland, G. D., et al. (2014). Biochemical and structural studies of NADH-dependent FabG used to increase the bacterial production of fatty acids under anaerobic conditions. Appl. Environ. Microbiol. 80, 497-505. doi: 10.1128/AEM.03194-13

Kellermann, M. Y., Schubotz, F., Elvert, M., Lipp, J. S., Birgel, D., PrietoMollar, X., et al. (2011). Symbiont-host relationships in chemosynthetic mussels: a comprehensive lipid biomarker study. Org. Geochem. 43, 112-124.

Kellermann, M. Y., Yoshinaga, M. Y., Valentine, R. C., Wörmer, L., and Valentine, D. L. (2016). Important roles for membrane lipids in haloarchaeal bioenergetics. Biochem. Biophys. Acta 1858, 2940-2956. doi.10.1016/j.bbamem.2016.08.010

Komen, R., Schmidt, K., and Friedrich, B. (1992). Hydrogenase mutants of Alcaligenes eutrophus $\mathrm{H} 16$ show alterations in the electron transport system. FEMS Microbiol. Lett. 96, 173-178. doi: 10.1111/j.1574-6968.1992.tb05412.x

Komen, R., Schmidt, K., and Zannoni, D. (1996). Hydrogen oxidation by membranes from autotrophically grown Alcaligenes eutrophus H16: role of the cyanide-resistant pathway in energy transduction. Arch. Microbiol. 165, 418-420. doi: 10.1007/s002030050347

Komen, R., Zannoni, D., Ingledew, W. J., and Schmidt, K. (1991a). The electron transport system of Alcaligenes eutrophus H16: I. Spectroscopic and thermodynamic properties. Arch. Microbiol. 155, 382-390. doi: 10.1007/ BF00243459

Komen, R., Zannoni, D., and Schmidt, K. (1991b). The electron transport system of Alcaligenes eutrophus H16: II. Respiratory activities and effect of specific inhibitors. Arch. Microbiol. 155, 436-443. doi: 10.1007/BF00244958

Kopf, S. H., Sessions, A. L., Cowley, E. S., Reyes, C., Van Sambeek, L., Hu, Y., et al. (2015). Trace incorporation of heavy water reveals slow and heterogeneous pathogen growth rates in cystic fibrosis sputum. Proc. Nat. Acad. Sci. U.S.A. 113, E110-E116. doi: 10.1073/pnas.1512057112, doi: 10.1073/pnas.1512057112

Kreuzer-Martin, H., Lott, M., Ehleringer, J. R., and Hegg, E. (2006). Metabolic processes account for the majority of the intracellular water in log-phase Escherichia coli cells as revealed by hydrogen isotopes. Biochemistry 45, 13622-13630. doi: 10.1021/bi0609164

Leavitt, W. D., Flynn, T. M., Suess, M. K., and Bradley, A. S. (2016). Transhydrogenase and growth substrate influence lipid hydrogen isotope ratios in Desulfovibrio alaskensis G20. Front. Microbiol. 7:918. doi: 10.3389/fmicb. 2016.00918.

Lee, J. N., Shin, H. D., and Lee, Y. H. (2003). Metabolic engineering of pentose phosphate pathway in Ralstonia eutropha for enhanced biosynthesis of polybeta-hydroxybutyrate. Biotechnol. Prog. 19, 1444-1449. doi: 10.1021/bp034060v

Lenz, O., and Friedrich, B. (1998). A novel multicomponent regulatory system mediates $\mathrm{H}_{2}$ sensing in Alcaligenes eutrophus. Proc. Natl. Acad. Sci. U.S.A. 95, 12474-12479. doi: 10.1073/pnas.95.21.12474

Osburn, M. R., Dawson, K. S., Fogel, M. L., and Sessions, A. L. (2016). Fractionation of hydrogen isotopes by sulfate- and nitrate-reducing bacteria. Front. Microbiol. 7:1166. doi: 10.3389/fmicb.2016.01166

Osburn, M. R., Sessions, A. L., Pepe-Ranney, C., and Spear, J. R. (2011). Hydrogen-isotopic variability in fatty acids from Yellowstone National Park hot spring microbial communities. Geochim. Cosmochim. Acta 75, 4830-4845. doi: 10.1016/j.gca.2011.05.038

Pfitzner, J. (1974). Contribution on H-O-oxidoreductase system of Hydrogenomonas eutropha strain H16 hydrogenase-defective mutants. Z. Bakteriol. Mikrobiol. Hyg. A 228, 121-127.

Sachse, D., Billault, I., Bowen, G. J., Chikaraishi, Y., Dawson, T. E., Feakins, S. J., et al. (2012). Molecular paleohydrology: interpreting the hydrogen-isotopic 
composition of lipid biomarkers from photosynthesizing organisms. Annu. Rev. Earth Planet. Sci. 40, 221-249. doi: 10.1146/annurev-earth-042711-105535

Schink, B., and Schlegel, H. G. (1978). Mutants of Alcaligenes eutrophus defective in autotrophic metabolism. Arch. Microbiol. 117, 123-129. doi: 10.1007/ BF00402299

Schink, B., and Schlegel, H. G. (1979). The membrane-bound hydrogenase of Alcaligenes eutrophus: 1. Solubilization, purification, and biochemical properties. Biochim. Biophys. Acta 567, 315-324. doi: 10.1016/0005-2744(79) 90117-7

Schneider, K., and Schlegel, H. G. (1976). Purification and properties of soluble hydrogenase from Alcaligenes eutrophus H16. Biochim. Biophys. Acta. 452, 66-80. doi: 10.1016/0005-2744(76)90058-9

Schouten, S., Ossebaar, J., Schreiber, K., Kienhuis, M. V. M., Langer, G., Benthien, A., et al. (2006). The effect of temperature, salinity and growth rate on the stable hydrogen isotopic composition of long chain alkenones produced by Emiliania huxleyi and Gephyrocapsa oceanica. Biogeosciences 3, 113-119. doi: 10.5194/bg-3-113-2006

Schwartz, E., Henne, A., Cramm, R., Eitinger, T., Friedrich, B., and Gottschalk, G. (2003). Complete nucleotide sequence of pHG1: a Ralstonia eutropha $\mathrm{H} 16$ megaplasmid encoding key enzymes of $\mathrm{H}_{2}$-based lithoautotrophy and anaerobiosis. J. Mol. Biol. 332, 369-383.

Sessions, A. L. (2006). Seasonal changes in D/H fractionation accompanying lipid biosynthesis in Spartina alternflora. Geochim. Cosmochim. Acta 70, 2153-2162. doi: 10.1016/j.gca.2006.02.003

Sessions, A. L., Burgoyne, T. W., Schimmelmann, A., and Hayes, J. M. (1999). Fractionation of hydrogen isotopes in lipid biosynthesis. Org. Geochem. 30, 1193-1200. doi: 10.1016/S0146-6380(99)00094-7

Sessions, A. L., and Hayes, J. M. (2005). Calculation of hydrogen isotopic fractionations in biogeochemical systems. Geochim. Cosmochim. Acta 69, 593-597. doi: 10.1016/j.gca.2004.08.005

Spencer, R. W., Daniels, L., Fulton, G., and Orme-Johnson, W. H. (1980). Product isotope effects on in vivo methanogenesis by Methanobacterium thermoautotrophicum. Biochemestry 19, 3678-3683. doi: 10.1021/bi00557a007

Tavormina, P. L., Kellermann, M. Y., Antony, C. P., Tocheva, E. I., Dalleska, N. F., Jensen, A. J., et al. (2017). Starvation and recovery in the deepsea methanotroph Methyloprofundus sedimenti. Mol. Microbiol. 103, 242-252. doi: $10.1111 / \mathrm{mmi} .13553$

Tejero, J., Peregrina, J. R., Martínez-Júlvez, M., Gutiérrez, A., Gómez-Moreno, C., Scrutton, N. S., et al. (2007). Catalytic mechanism of hydride transfer between $\mathrm{NADP}+/ \mathrm{H}$ and ferredoxin-NADP+ reductase from Anabaena PCC 7119. Arch. Biochem. Biophys. 459, 79-90.

Valentine, D. L., Sessions, A. L., Tyler, S. C., and Chidthaisong, A. (2004). Hydrogen isotope fractionation during $\mathrm{H}_{2} / \mathrm{CO}_{2}$ acetogenesis: hydrogen utilization efficiency and the origin of lipid-bound hydrogen. Geobiology 2, 179-188. doi: $10.1111 / j .1472-4677.2004 .00030 . x$
Vandamme, P., and Coenye, T. (2004). Taxonomy of the genus Cupriavidus: a tale of lost and found. Int. J. System. Evol. Microbiol. 54, 2285-2289. doi: 10.1099/ijs. $0.63247-0$

Vaneechoutte, M., Kampfer, P., De Baere, T., Falsen, E., and Verschraegen, G. (2004). Wautersia gen. nov., a novel genus accommodating the phylogenetic lineage including Ralstonia eutropha and related species, and proposal of Ralstonia [Pseudomonas] syzygii (Roberts et al. 1990) comb. nov. Int. J. Syst. Evol. Microbiol. 54, 317-327. doi: 10.1099/ijs.0.02754-0

Vignais, P. M. (2005). H/D exchange reactions and mechanistic aspects of the hydrogenases. Coord. Chem. Rev. 249, 1677-1690. doi: 10.1016/j.ccr.2005. 01.026

Vignais, P. M., and Colbeau, A. (2004). Molecular biology of microbial hydrogenases. Curr. Issues Mol. Biol. 6, 159-188.

Wörmer, L., Lipp, J. S., Schröder, J. M., Hinrichs, K.-U. (2013). Application of two new LC-ESI-MS methods for improved detection of intact polar lipids (IPLs) in environmental samples. Org. Geochem. 59, 10-21. 10.1016/j.orggeochem.2013.03.004

Yabuuchi, E., Kosako, Y., Yano, I., Hotta, H., and Nishiuchi, Y. (1995). Transfer of two Burkholderia and an Alcaligenes species to Ralstonia gen. nov.: Proposal of Ralstonia pickettii (Ralston, Palleroni and Doudoroff 1973) comb. nov., Ralstonia solanacearum (Smith 1896) comb. nov. and Ralstonia eutropha (Davis 1969) comb. nov. Microbiol. Immunol. 39, 897-904. doi: 10.1111/j.1348-0421. 1995.tb03275.x

Zhang, X., Gillespie, A. L., and Sessions, A. L. (2009a). Large D/H variations in bacterial lipids reflect central metabolic pathways. Proc. Natl. Acad. Sci. U.S.A. 106, 12580-12586. doi: 10.1073/pnas.09030 30106

Zhang, Z., Sachs, J. P., and Marchetti, A. (2009b). Hydrogen isotope fractionation in freshwater and marine algae: II. Temperature and nitrogen limited growth rate effects. Org. Geochem. 40, 428-439. doi: 10.1016/j.orggeochem.2008.11.002

Zhang, Z., and Sachs, J. P. (2007). Hydrogen isotope fractionation in freshwater algae: I. Variations among lipids and species. Org. Geochem. 38, 582-608. doi: 10.1016/j.orggeochem.2006.12.004

Conflict of Interest Statement: The authors declare that the research was conducted in the absence of any commercial or financial relationships that could be construed as a potential conflict of interest.

Copyright $\odot 2017$ Campbell, Sessions, Fox, Paul, Qin, Kellermann and Valentine. This is an open-access article distributed under the terms of the Creative Commons Attribution License (CC BY). The use, distribution or reproduction in other forums is permitted, provided the original author(s) or licensor are credited and that the original publication in this journal is cited, in accordance with accepted academic practice. No use, distribution or reproduction is permitted which does not comply with these terms. 